Cahiers « Mondes anciens »

ANCIENS

Histoire et anthropologie des mondes anciens

$7 \mid 2015$

Marchands romains au long cours

Amphores italiques en Lycie : témoins des réseaux marchands en Méditerranée orientale ? (II ${ }^{\mathrm{e}}$ s. av. J.-C./I ${ }^{\text {er }}$ s. ap. J.-C.)

Italian Amphoras in Lycia: Indices of the Trade Networks in the Eastern

Mediterranean Sea (IInd century BC / Ist century AD).

\title{
Séverine Lemaître
}

\section{(2) OpenEdition}

Journals

Édition électronique

URL : http://journals.openedition.org/mondesanciens/1617

DOI : 10.4000/mondesanciens. 1617

ISSN : 2107-0199

Éditeur

UMR 8210 Anthropologie et Histoire des Mondes Antiques

Référence électronique

Séverine Lemaître, «Amphores italiques en Lycie : témoins des réseaux marchands en Méditerranée orientale? (I" s. av. J.-C.///er s. ap. J.-C.) », Cahiers « Mondes anciens » [En ligne], 7| 2015, mis en ligne le 21 décembre 2015, consulté le 05 mai 2019. URL : http://journals.openedition.org/ mondesanciens/1617; DOI : 10.4000/mondesanciens.1617

Ce document a été généré automatiquement le 5 mai 2019.

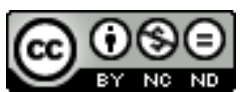

Les Cahiers "Mondes Anciens " sont mis à disposition selon les termes de la licence Creative Commons Attribution - Pas d'Utilisation Commerciale - Pas de Modification 4.0 International. 


\title{
Amphores italiques en Lycie : témoins des réseaux marchands en Méditerranée orientale? (II S. av. J.-C./I
}

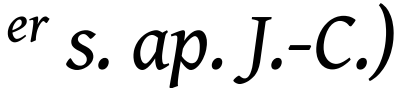

\author{
Italian Amphoras in Lycia: Indices of the Trade Networks in the Eastern \\ Mediterranean Sea (IInd century BC / Ist century AD).
}

\section{Séverine Lemaître}

1 Les rencontres initiées à Poitiers par Nicolas Tran proposaient d'interroger l'hypothèse de l'existence d'élites marchandes dans la société romaine antique selon des modèles définis pour la période moderne. La connaissance de ces groupes sociaux passent par l'étude de leur identité et de l'origine de leurs richesses, celles-ci étant issues au moins en partie de revenus tirés de l'activité commerciale. Ce que nous appelons «activité commerciale » correspond ici à ce qui a été désigné sous le nom de grand commerce, dans le sens où il met en jeu des circulations de marchandises sur de moyennes et longues distances, essentiellement par voie maritime, ainsi que des capitaux importants dans le cadre de réseaux d'échanges complexes et inter-dépendants.

2 La contribution des amphores à la connaissance des activités de productions, essentiellement agricoles et des réseaux commerciaux en œuvre en Méditerranée à la fin de la période républicaine n'est plus à démontrer (Tchernia 1986a). Les emballages que sont les amphores ne dévoilent que rarement, dans le cas d'estampilles, le nom de personnes en lien avec leur production. De plus, J.-Y. Empereur et d'autres ont largement attiré l'attention sur la faible part que pouvaient représenter les conteneurs timbrés par rapport à l'ensemble des productions d'amphores antiques et le poids « réel » de certaines productions dans les échanges selon la fréquence plus ou moins grande du timbrage (Empereur 1982, p. 226). Parallèlement, les inscriptions peintes, en révélant des noms de marchands, fournissent des éléments sur la commercialisation du produit qu'elles 
transportaient. Mais ces documents épigraphiques, précieux pour la connaissance des réseaux en oeuvre en Méditerranée, sont mal conservés sur les conteneurs découverts le plus souvent dans les poubelles des sites terrestres antiques, une fois le produit qu'elles transportaient, transvasé et consommé.

Les amphores ne permettent pas d'aborder, sauf à la marge en cas de timbrage ou d'inscription peinte, les questions d'ordre sociologique proposées en introduction à la rencontre de Poitiers. Toutefois, les recherches que nous avons menées sur les amphores découvertes en différents points de l'espace méditerranéen, apportent un éclairage sur un des aspects évoqués par N. Tran, lors de ces journées, à savoir l'expansion du grand commerce du vin et de l'huile italiens à la fin de la période républicaine.

\section{Historique des recherches sur la diffusion des amphores italiques en Orient}

Les amphores et leur lien avec la production et la commercialisation du vin notamment, ont déjà été utilisés pour définir partiellement la nature des intérêts italiens en Méditerranée orientale (Hatzfeld 1919, p.144-145). Les découvertes de conteneurs d'origine italique dans ces régions a suscité de nombreux travaux qui ont tous en commun un essai d'évaluation du poids ou de la part que représentent les exportations italiques dans l'économie de ces régions (voir sur ce point Lawall 2006). Pour E. Lyding Will, les découvertes d'amphores italiques en Égée sont suffisantes en nombre pour parler d'une politique délibérée de domination économique menée dans cette région par les Romains (Will 1989, p. 298 puis Will 1997). N. Rauh s'interroge lui aussi sur la signification de la présence d'amphores italiques en Méditerranée orientale (Rauh 2003, p. 127). À la suite de M. Rostovtzeff, cité également par A. Tchernia (Tchernia 1986a, p.71-72), il soutient plutôt l'hypothèse que les découvertes d'amphores italiques en Méditerranée orientale reflètent la consommation des Italiens installés dans ces régions (Rauh 1999). Toutefois, il rejoint aussi la position d'E. Lyding Will car il écrit que l'histoire de la conquête romaine du monde méditerranéen est aussi l'histoire de la domination romaine sur le commerce maritime (Rauh 2003, p. 133). À l'inverse, J. Lund insiste sur le caractère très inégal des données quantitatives à propos du matériel romain mis au jour en différents points du bassin oriental de la Méditerranée et avertit sur le caractère prématuré d'une conclusion à propos de la suprématie économique de Rome dans ces régions dès le milieu du $\mathrm{II}^{\mathrm{e}}$ siècle av. J.-C. (Lund 2000, p. 89). En effet, les publications de plus en plus nombreuses d'ensembles d'amphores, à Pergame, Argos, Nea Paphos, Halicarnasse, Alexandrie (şenol 2007, p. 61-62), Éphèse (Bezeczky 2004, p. 85 et Bezeczky 2006, p. 289-291) ou au Levant sud (Finkielsztejn 2001), tendent à présenter une situation contrastée face à la réception des denrées commercialisées dans les amphores italiques (Lund 2000, p. 88-89 et p. 99 pour les références bibliographiques associées aux autres sites mentionnés). Une des difficultés essentielles de l'évaluation de la part des amphores italiques dans l'économie tient au fait que, pendant des décennies, les chercheurs ont étudié ces conteneurs pour eux-mêmes, de manière isolée, sans tenir compte de leur contexte de découverte ni de leur place au sein d'un assemblage d'amphores. Les données quantitatives globales mériteraient donc d'être étoffées.

5 Loin de prétendre aborder un sujet vierge, notre présentation s'inscrit dans la continuité d'une démarche initiée par M. Lawall à propos des amphores italiques en Égée (Lawall 
2006). En évitant l'écueil que constitue le commentaire du nombre d'amphores italiques découvertes en Méditerranée orientale, $M$. Lawall propose en effet un nouveau modèle d'interprétation fondé sur l'observation des économies régionales selon trois variables : le niveau relatif et la complexité des productions orientales, l'inter-dépendance des réseaux de circulation régionaux des amphores et la dépendance par rapport aux réseaux d'importations d'amphores à longue distance. Ce modèle présuppose l'idée que l'utilisation des amphores pour commercialiser des produits agricoles était un phénomène courant, sachant que l'agriculture était une des sources principales de revenus dans l'Antiquité (Lawall 2006, p. 266).

Such an approach does not deny the importance of changes, developments, perhaps even deliberate policy in the western Mediterranean, but it seeks to balance such studies of late Republican Italian wine and oil exports with the perspective of the affected regions (Lawall 2006, p. 267).

6 L'élément le plus original dans cette approche est que l'auteur prend comme point de départ des assemblages de mobilier amphorique provenant de sites de consommation localisés dans différentes régions de la mer Égée. Il privilégie des ensembles bien stratigraphiés ayant livré des quantités suffisantes de tessons et il définit ce que nous appelons des faciès d'approvisionnement pour chaque région envisagée dans un cadre chronologique resserré, en précisant à chaque fois la part des amphores nord-égéennes, sud-égéennes ou occidentales, en y associant des données typologiques et quantitatives. Précisons que cette démarche est celle mise en œuvre régulièrement pour la Gaule celtique puis romaine afin d'apprécier la réception des importations méditerranéennes et les phénomènes d'acculturation induits par la consommation de denrées nouvelles transportées en amphore, comme le vin, l'huile d'olive ou les sauces et conserves de poisson, dans la moitié nord de la Gaule par exemple (entre autres Baudoux 1996, Poux 2004, Laubenheimer et Marlière 2010, Guitton et Lemaître 2012, Lemaître 2014).

7 Les faciès d'approvisionnement définis sont ensuite replacés par M. Lawall sur le temps long afin de mieux saisir les changements intervenus dans le monde égéen depuis la fin de l'époque archaïque. L'auteur parvient ainsi à mettre en lumière les pulsations intervenues dans les économies des régions égéennes. Il emploie le terme d'« élasticité » plus ou moins grande à propos de la capacité des économies régionales égéennes à absorber notamment les exportations occidentales (Lawall 2006, p. 267).

Son enquête aboutit, de manière convaincante, à la définition de régions (de taille très différente) aux comportements spécifiques et changeant au fil du temps: le nord de l'Égée, le sud-est de l'Égée, Athènes et ce qu'il appelle la "région adriatique" correspondant à l'ouest de la Grèce propre. Corinthe constitue le point oriental extrême de cette zone, qui géographiquement est la plus propice à recevoir les marchandises expédiées non seulement depuis la façade adriatique de la péninsule italienne, mais également de la côte tyrrhénienne, ce qui se vérifie lors de l'examen des tessons d'amphores depuis l'époque classique (Lawall 2006, p. 268 et 270).

9 L'analyse que nous avons menée sur plusieurs ensembles d'amphores découverts en Gaule mais également en Orient, en Lycie, à Beyrouth et plus récemment à Alexandrie est à l'origine de réflexions à propos de l'organisation des réseaux commerciaux antiques à partir de la péninsule italienne. Les rencontres de Poitiers ont été l'occasion pour nous de présenter un des aspects d'un travail de recherche archéologique engagé sur la diffusion des productions italiques et plus spécifiquement campaniennes en Méditerranée. 
Fig. 1 : Carte de localisation de la Lycie antique et des sites mentionnés dans le texte.

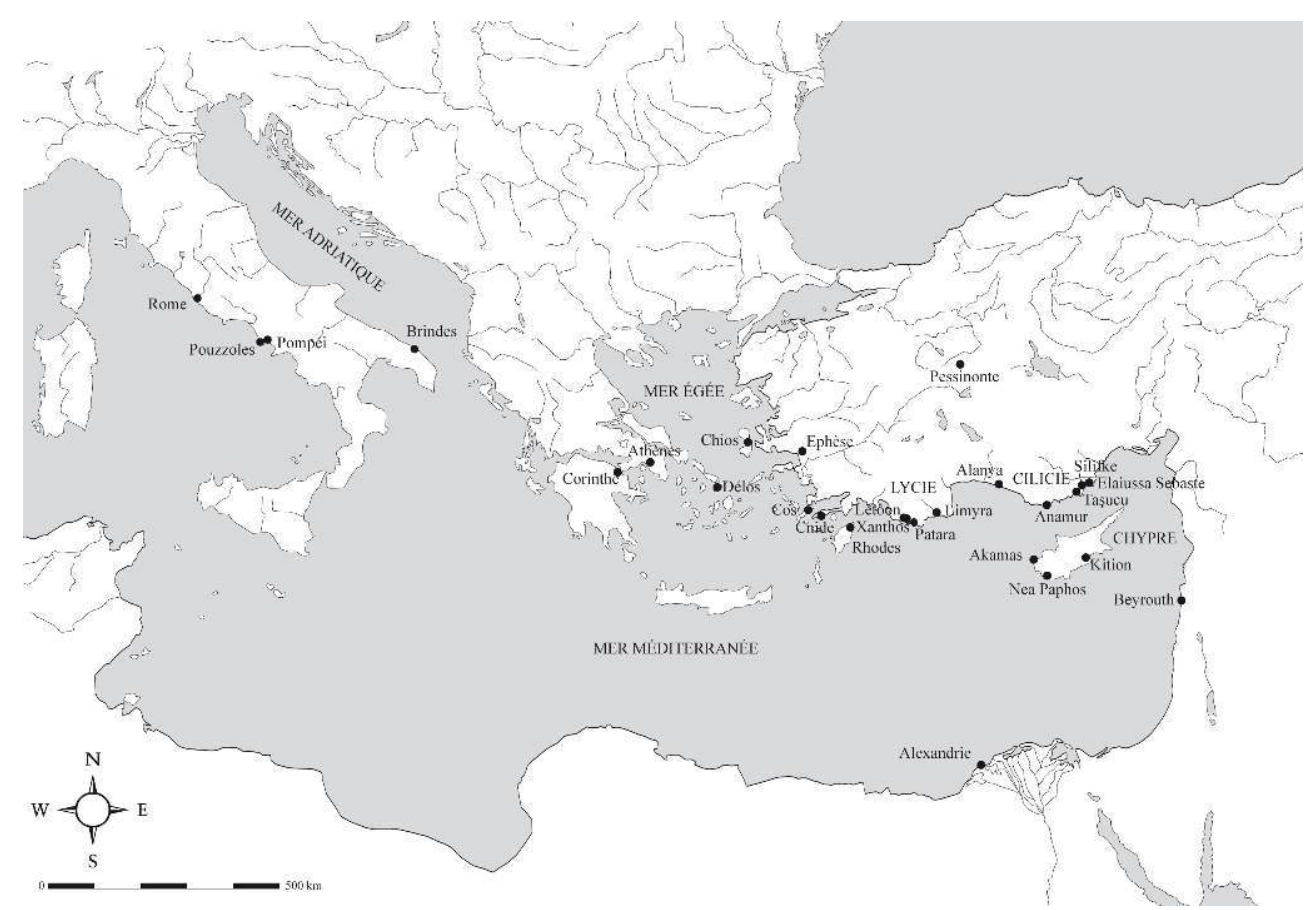

Précisément, notre contribution consiste avant tout ici, en un complément de la carte de découverte des produits italiques en Méditerranée orientale dans une région encore assez mal documentée du point de vue des amphores, même si les recherches ont pris ces dernières années un essor certain grâce en particulier aux travaux d'E. Dündar (fig. 1). Les découvertes faites à Xanthos en Lycie offrent l'opportunité de nous inscrire dans le débat concernant les exportations d'amphores italiques en Orient en adoptant, à une échelle plus modeste, la démarche initiée par M. Lawall.

11 Il convient auparavant de revenir rapidement sur quelques éléments de typologie amphorique liés à différentes régions de productions italiques et dont les conteneurs ont été commercialisés, à des degrés divers, dans l'ensemble de l'espace méditerranéen.

\section{Les amphores produites en Italie à la fin de la République et au début de l'Empire}

L'efficacité et la pertinence des études sur les amphores résident dans l'identification stricte des types rencontrés dans les sites de consommation et la définition la plus précise possible des origines des conteneurs grâce notamment à l'observation des pâtes argileuses avec lesquelles ils ont été façonnés. Rappelons que ce dernier point est une des clés pour tenter de reconstituer les réseaux de commercialisation de certaines denrées. Parmi les produits italiques transportés en amphores, le vin est très largement prépondérant, même si l'huile de la région de Brindes a fait l'objet d'une commercialisation relativement importante vers l'Orient (Will 1989).

13 À ces deux denrées principales, peut-être faudrait-il ajouter les sauces et les conserves préparées à base de poisson. En effet, la production et la commercialisation de salaisons et de sauces de poissons en Italie du sud et en Sicile ont été récemment mises en lumière 
par les travaux d'E. Botte (Botte 2009). Sans pouvoir assigner de manière absolue un produit fabriqué à base de poisson aux amphores "puniques » produites en Sicile, mais après avoir examiné les types d'amphores découverts dans les usines à salaison siciliennes, E. Botte émet l'hypothèse : «... que ces amphores étaient destinées au transport de sauces et/ou salaisons de poisson...» (Botte 2009, p. 115). Des exportations vers l'Orient ont existé au moins au $\mathrm{III}^{\mathrm{e}}$ siècle av. J.-C. L'auteur rappelle la mention d'un envoi de dix mille jarres de salaisons siciliennes à Alexandrie par Hiéron II, tyran de Syracuse (Athénée, Deipn., V, 11, 209a cité dans Botte 2009, p. 116). Une mention littéraire ne fait pas un réseau commercial, mais étant donné que la production d'amphores a perduré jusqu'au Haut Empire, notamment sous les formes Dressel 21/22 et que ces conteneurs sont attestés en Méditerranée orientale en plusieurs points, à Éphèse (Bezeczky 2010), Alexandrie (şenol 2007, p. 65, diag. 2), Beyrouth (Lemaître 2007a, p. 281-282), en Lycie, dans le sanctuaire du Létôon (Lemaître, Élaigne et Alary 2004, p. 333) ou encore dans les collections du musée d'archéologie sous-marine de Bodrum (Alpözen et alii 1995, p. 102, $\mathrm{n}^{\circ} 4245$ ), on peut légitimement proposer que des exportations de salaisons italiques et/ou siciliennes existaient en direction du bassin oriental de la Méditerranée entre la fin de la République et l'époque impériale.

Fig. 2 : Amphores italiques des $\|^{\mathrm{e}}$ et $\mathrm{I}^{\mathrm{er}}$ siècles av. notre ère et du début du $\mathrm{I}^{\mathrm{er}}$ siècle commercialisées en Méditerranée orientale.
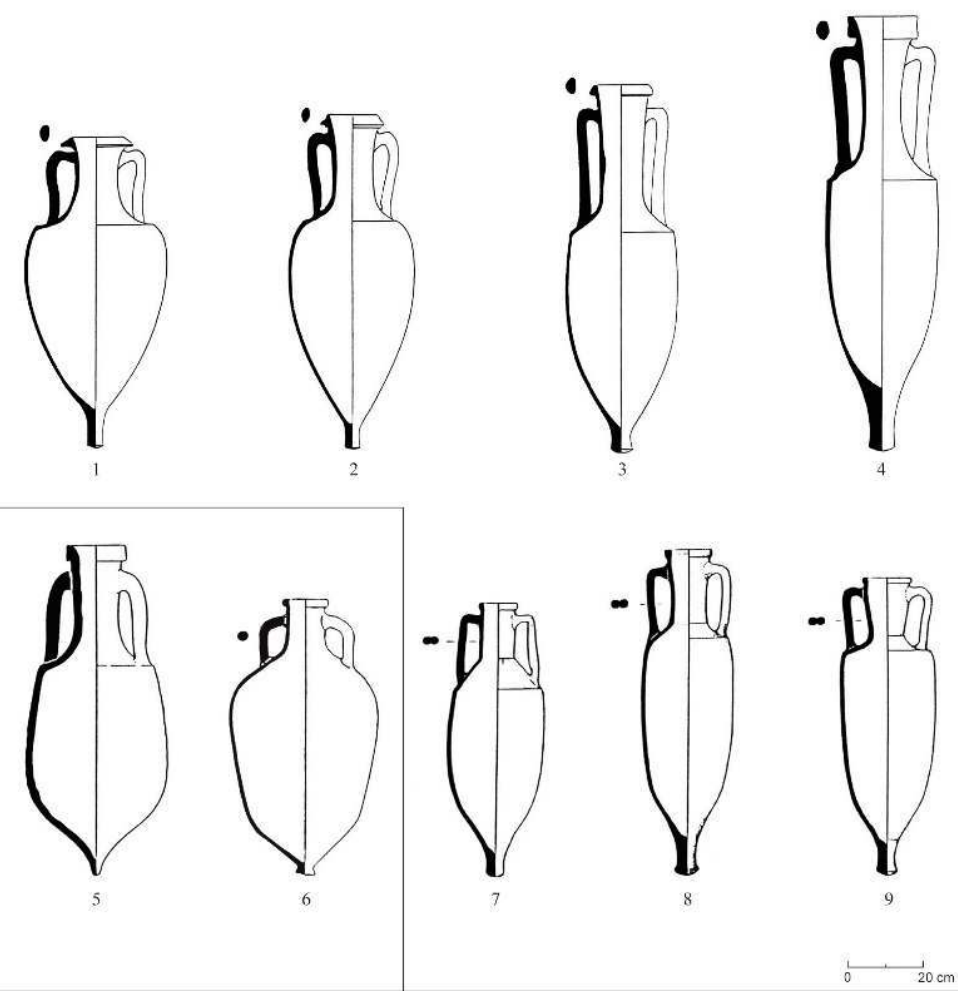

Le tableau typologique et quantitatif des exportations italiques en Méditerranée orientale a été réalisé il y a quelques années par E. Lyding Will (Will 1989), puis par J. Lund (Lund 2000), complété par M. Lawall (Lawall 2006), puis T. Bezeczky plus spécifiquement pour Éphèse (Bezeczky 2008). Nous ne reprenons ici que les grandes lignes de ces études, complétées par des publications plus récentes. 

façade adriatique, signalées à Athènes et à Corinthe à la fin $\mathrm{du} \mathrm{VI}^{\mathrm{e}}$ siècle et durant le siècle suivant (Lawall 2006, p. 267-269), les amphores italiques les plus précocement importées en Orient sont les amphores dites gréco-italiques (Will Type 1a), lointaines héritières de modèles grecs de l'époque classique et dont la production, centrée dans le sud de l'Italie, est datée entre le dernier tiers du $\mathrm{IV}^{\mathrm{e}}$ et le premier tiers du $\mathrm{III}^{\mathrm{e}}$ siècle av. J.-C. (Will 1982, Lund 2000, p. 78-79). J. Lund indique que ces amphores ont été découvertes au sein du monde grec dans des territoires, à cette époque sous domination lagide (Lund 2000, p. 80).

\section{- Amphore gréco-italique et Dressel 1}

Dans la deuxième moitié du ${ }^{\mathrm{III}}{ }^{\mathrm{e}}$ siècle et dans la première moitié du siècle suivant, la morphologie des amphores gréco-italiques destinées à la commercialisation du vin de la côte tyrrhénienne évolue sensiblement (fig. 2,1). La silhouette du conteneur s'étire et le diamètre de la panse diminue. La forme est dotée d'un pied cylindrique, haut et fin, et le bord est caractérisé par une lèvre de section triangulaire aplatie. Produite dans l'ager Cosanus, en Campanie, en Lucanie, dans le Bruttium et en Sicile (Tchernia 1986a, p. 48 et Vandermersch 1994 cité par J. Lund), elle connaît une diffusion plus large en Méditerranée orientale et en faibles quantités, incluant la Grèce, (Lawall 2006, p. 270), la Turquie, Chypre, Israël, l'Égypte et la Libye (Lund 2000, p. 80).

Sur la côte tyrrhénienne les productions gréco-italiques cèdent la place aux amphores Dressel 1 vers 150 av. J.-C. Dans une première phase on peut parler d'amphores de transition entre gréco-italiques et Dressel $1 \mathrm{~A}$, encore caractérisées par une lèvre de section triangulaire mais avec une nette tendance à l'allongement de la panse et du col (fig. 2, 2). Les dernières décennies $d u$ II ${ }^{e}$ siècle et les premières décennies du siècle suivant correspondent à la période de production des amphores Dressel 1A à lèvre triangulaire ou en bandeau court (fig. 2, 3), dont la hauteur augmente dans le courant du $\mathrm{I}^{\mathrm{er}}$ siècle avant notre ère, aboutissant au type Dressel 1B (fig. 2, 4). Ce dernier modèle est marqué par sa robustesse : une embouchure large, un pied haut et massif, des anses longues ainsi qu'une panse à la paroi épaisse. Aux côtés des productions de l'Adriatique, les amphores Dressel 1 sont désormais attestées dans tous les grands sites urbains du bassin oriental de la Méditerranée, en Grèce et au Levant (Lund 2000, p. 82-83). À Athènes, également touchée par ces exportations, M. Lawall insiste sur la présence dans les assemblages d'amphores italiques de différentes fabrics, d'Étrurie, de Campanie mais également d'autres indéterminées pour l'instant (Lawall 2006, p. 272). Il est intéressant de noter que cette diversité des pâtes argileuses des amphores italiques est aussi largement perceptible dans les ensembles des $\mathrm{II}^{\mathrm{e}}$ et $\mathrm{I}^{\mathrm{er}}$ siècles avant notre ère découverts sur le territoire gaulois (Olmer 2003, p. 188-192). Cette diversité est à mettre en relation avec la dispersion des ateliers de production d'amphores sur la côte tyrrhénienne et le long des vallées que F. Olmer a regroupés en quatre zones principales : l'ager Pisanus et l'ager Volateranus; l' ager Cosanus ; l'Étrurie méridionale et le Latium et la Campanie (Olmer 2003, p. 191 carte 49).

Étrurie et Campanie voient également la production d'une variante appelée Dressel $1 \mathrm{C}$ à la fin $d u$ II $^{\mathrm{e}}$ et dans la première moitié du $\mathrm{I}^{\mathrm{er}}$ siècle avant notre ère. Elle est caractérisée par une embouchure rentrante et des anses au profil sinueux marquées par des nervures assez nettes (Empereur et Hesnard 1987, p. 31-32). 
19 Le type d'amphore Dressel 1, diffusé très largement et massivement vers la Gaule, un peu moins vers l'Orient, est à son tour quasiment abandonné vers $50 \mathrm{av}$. J.-C. (Desbat 1998) ou peu après dans les années 40-30 av. J.-C. Comme l'amphore Dressel 1A, la forme ultime des conteneurs à vin républicains de la côte tyrrhénienne a été identifiée dans peu de régions du bassin oriental de la Méditerranée : en Turquie à Éphèse (Bezeczky 2006, p. 288, sous forme résiduelle dans un ensemble daté du $\mathrm{I}^{\mathrm{er}}$ siècle ap. J.-C.). Dans la ville, les amphores Dressel 1 sont notamment attestées parmi les mobiliers archéologiques des fouilles des différents niveaux d'une rue située devant la stoa d'époque hellénistique (Bezeczky 2004, p. 85).

20 En Égypte, elles sont connues à Alexandrie (Empereur 1998, p. 394 et şenol 2007) mais aussi dans le delta oriental du Nil dans le site de Bouto (Lemaître, étude inédite de mobilier de prospection 2012, dir. P. Ballet) et Lemaître (à paraître). À Athènes globalement les importations italiques ont été plus nombreuses que dans le sud-est égéen notamment à partir de la fin du II ${ }^{\mathrm{e}}$ siècle av. J.-C. (Lawall 2006, p. 276).

\section{- Amphore Lamboglia 2}

21 Sur la façade adriatique, le II ${ }^{\mathrm{e}}$ siècle coïncide avec la période de production des amphores de type Lamboglia 2 fabriquées dans plusieurs ateliers installés dans la partie centrale et septentrionale de la côte adriatique, maintenant mieux connus grâce à l'étude de dépotoirs liés à des zones de production (Cipriano et Carre 1989, p. 83 et plus récemment à propos du Picenum méridional Menchelli et al. 2008). Ce conteneur destiné au transport du vin (Formenti, Hesnard et Tchernia 1978), de forme trapue présente une lèvre en bandeau court, une panse ovoïde aux parois épaisses se terminant par une pointe effilée. Les anses robustes sont de section ronde (fig. 2, 5). Bien que variées, les argiles avec lesquelles ces amphores ont été façonnées sont reconnaissables. Fines, de couleur rose ou orange, certaines d'entre elles contiennent des nodules argileux de taille irrégulières, tantôt plus clairs, tantôt plus foncés que la matrice. Elles ont été massivement diffusées vers les grands sites urbains orientaux aux $\mathrm{II}^{\mathrm{e}}-\mathrm{I}^{\mathrm{er}} \mathrm{s}$. av. J.-C., tandis que leur commercialisation vers l'ouest et la Gaule en particulier, a été bien moindre (Tchernia 1986a, p.68; Empereur et Hesnard 1987, p. 33 ; Lund 2000, p. 84). Elles sont particulièrement nombreuses à Délos (Empereur et al. 1983, p. 882 ; Empereur 1983, p. 98).

Vers 30 av. J.-C. les amphores Lamboglia 2 évoluent vers le type Dressel 6 (Tchernia 1986a, p.134), qui reste assez proche de l'ancien modèle et dont la production se poursuit à l'époque impériale (Cipriano et Carre 1989, p. 84). Cette forme évoluée apparaît de manière sporadique en Méditerranée orientale à Éphèse (Bezeczky 2004, p. 86, p. 88-89) et Alexandrie, dans la fouille du théâtre Diana (şenol 2007, p. 71). Les données quantitatives disponibles semblent montrer que les amphores à vin Lamboglia 2 de la côte adriatique étaient beaucoup plus nombreuses à Athènes et Délos (Will 1997, p.123-126) qu'à Alexandrie (Empereur 1982, p. 110).

\section{- Amphore ovoïde de l'Adriatique}

La côte adriatique a produit également en Apulie et à Brindes notamment, des amphores sans doute destinées à la commercialisation de l'huile d'olive (Cipriano et Carre 1989). Ces conteneurs appartiennent à une famille d'amphores ovoïdes, dont une série porte la célèbre estampille M. Tuccius Galeo (Cipriano et Carre 1989, p. 75-77) ${ }^{1}$. Leur forme est assez 
reconnaissable même si le traitement de la lèvre a évolué sensiblement au fil du temps, présentant tantôt un bandeau, tantôt un bourrelet épais (fig. 2, 6). Les anses sont courbes et de section ronde, tandis que la panse se referme en un petit pied court, plus ou moins large. La chronologie de la production se place entre la fin du II siècle avant notre ère et la période augustéenne (Empereur et Hesnard 1987, p. 34). Ces formes ont aussi été fabriquées dans des ateliers du sud du Latium (Empereur et Hesnard 1987, p. 35). La recension menée par J.Lund sur les occurrences de ces conteneurs en Méditerranée orientale indique qu'elles y ont été largement commercialisées, plus précisément en Grèce, Turquie, Chypre, Syrie, Israël, Égypte et Libye (Lund 2000, p. 84-85 et p. 98 pour les références aux sites de découverte). Il faut signaler la présence d'au moins un conteneur de Brindes dans les collections du musée d'Anamur sur la côte sud anatolienne².

\section{- Amphore Dressel 2/4} sociales, économiques et politiques (Andreau 1997), peut-être aussi à l'origine de mutations dans la morphologie des conteneurs destinés à la commercialisation des vins italiques. (Empereur et Hesnard 1987, p. 22-23) correspond aux amphores de forme Dressel 2, 3 et 4 de la table typologique du savant allemand (Dressel 1899). La forme générale de l'amphore est cylindrique, marquée par un épaulement oblique. La panse se termine par un petit pied court sur les productions les plus anciennes. Les anses ont la particularité d'être bifides et la lèvre est bourrelet assez fin (fig. 2, 7, 8 et 9).

Ce type fait son apparition dans les ateliers producteurs de Dressel 1 de la façade tyrrhénienne de l'Italie à partir du milieu du $\mathrm{I}^{\mathrm{er}}$ siècle av. J.-C. (Hesnard 1977, Hesnard et Lemoine 1981). Sa fabrication s'est poursuivie, sous une forme évoluée au moins jusque dans le courant du $\mathrm{II}^{\mathrm{e}}$ siècle de notre ère. En témoigne sa commercialisation dans la vallée du Rhône au II ${ }^{\mathrm{e}}$ siècle et peut-être encore au début du III ${ }^{\mathrm{e}}$ siècle ap. J.-C. (Desbat et SavayGuerraz 1990, p. 211). Des amphores Dressel 2/4 ont aussi été fabriquées sur la façade adriatique, mais leur diffusion reste encore peu connue (Empereur et Hesnard 1987, p. 36).

Ce changement radical de modèle de récipient pour la commercialisation du vin italique en amphores a fait l'objet de discussions fournies de la part des spécialistes (Hesnard 1977, p. 162 et Manacorda 1981, p. 25 ; Tchernia 1986a ou 1986b, p. 135). A. Hesnard a mis en évidence le fait que l'amphore Dressel 2/4 permettait, grâce à sa morphologie combinée aux techniques de chargement à bord des navires, de transporter davantage de vin en un voyage. " À titre d'exemple, un rapide calcul (selon la méthode utilisée pour la cargaison de Dr. 1) montre que le volume de cargaison occupé par 4500 Dr. 1 peut contenir plus de 6000 Dr. 2-4, soit un gain de $30 \%$ de vin transporté par un même navire " (Hesnard 1977, p. 162, note 28). Ce gain est lié à certaines caractéristiques techniques propres aux amphores de Cos, le modèle imité par les potiers italiens, à savoir des parois beaucoup plus minces et des éléments rajoutés à la panse (col, anses et surtout pieds) bien moins massifs et lourds que sur les amphores de type Dressel 1. A. Tchernia a mis en lumière un passage du livre XXXV de l'Histoire Naturelle de Pline, dans lequel il est question de la tenuitas des amphores de Cos, opposée à la firmitas des amphores d'Hadria (Tchernia 1986b, p. 31-34).

Cahiers « Mondes anciens », 7| 2015 

d'amphore, qui a été imitée non seulement en Méditerranée orientale mais également très largement, à partir de l'époque augustéenne, dans les provinces occidentales de l'Empire, de l'Égypte au sud de la Grande-Bretagne ! Les imitations ont été plus ou moins fidèles au modèle initial, ce qui permet de distinguer, dans la plupart des cas, en l'associant à l'observation macroscopique des pâtes argileuses, la région d'origine des productions. Toutefois le recours à des analyses pétrographiques et/ou physicochimiques est indispensable dans certains cas pour préciser l'origine des tessons, notamment au sein des grandes régions de production.

Les amphores Dressel 2/4 italiques ont connu une diffusion large en Méditerranée orientale et au-delà jusque dans la péninsule indienne (Williams 2004), mais relativement limitée d'un point de vue quantitatif. Elles ont été identifiées entre autres à Athènes, Éphèse (Bezeczky 2010), Beyrouth (Lemaître 2007, p. 281), Alexandrie (şenol 2007 et Lemaître étude inédite), et dans le Nord Sinai (Arthur et Oren 1998, p. 197).

Les recherches que nous avons menées en Lycie $^{3}$ permettent aujourd'hui d'envisager cette région comme terrain d'étude de la réception des exportations italiques même si, nous le verrons, celles-ci apparaissent en quantité limitée.

\section{Des amphores italiques en Lycie}

31

Outre l'opportunité d'y avoir travaillé pendant plusieurs années, le choix de la Lycie s'explique par la place particulière qu'occupe cette région du sud de l'Anatolie dans l'organisation des routes qui parcourent le bassin oriental de la Méditerranée.

\section{- Découvertes à Xanthos}


Fig. 3 : Xanthos, agora supérieure et basilique civile, localisation du sondage 14050.

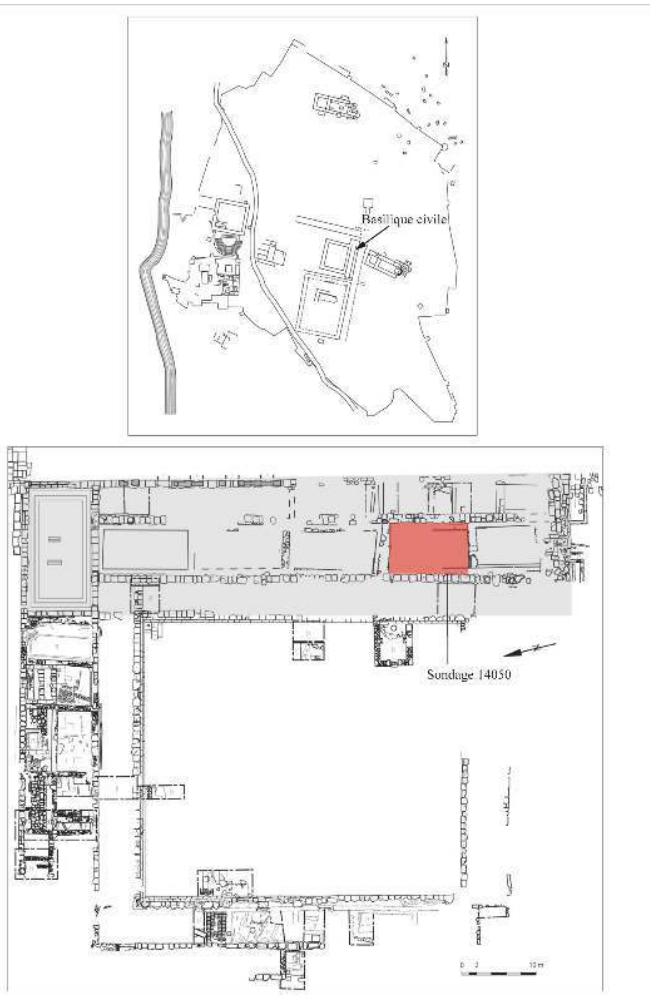

D'après Cavalier 2012, p. 189 et p. 190, fig. 2 (relevés : M.-G. Froidevaux, F. Goirand et E. Cayre ; DAO E. Cayre ; Topographie : R. Bernard). 
Fig. 4 : Xanthos, agora supérieure, basilique civile, coupe stratigraphique du sondage 14050. Localisation du remblai 14055.

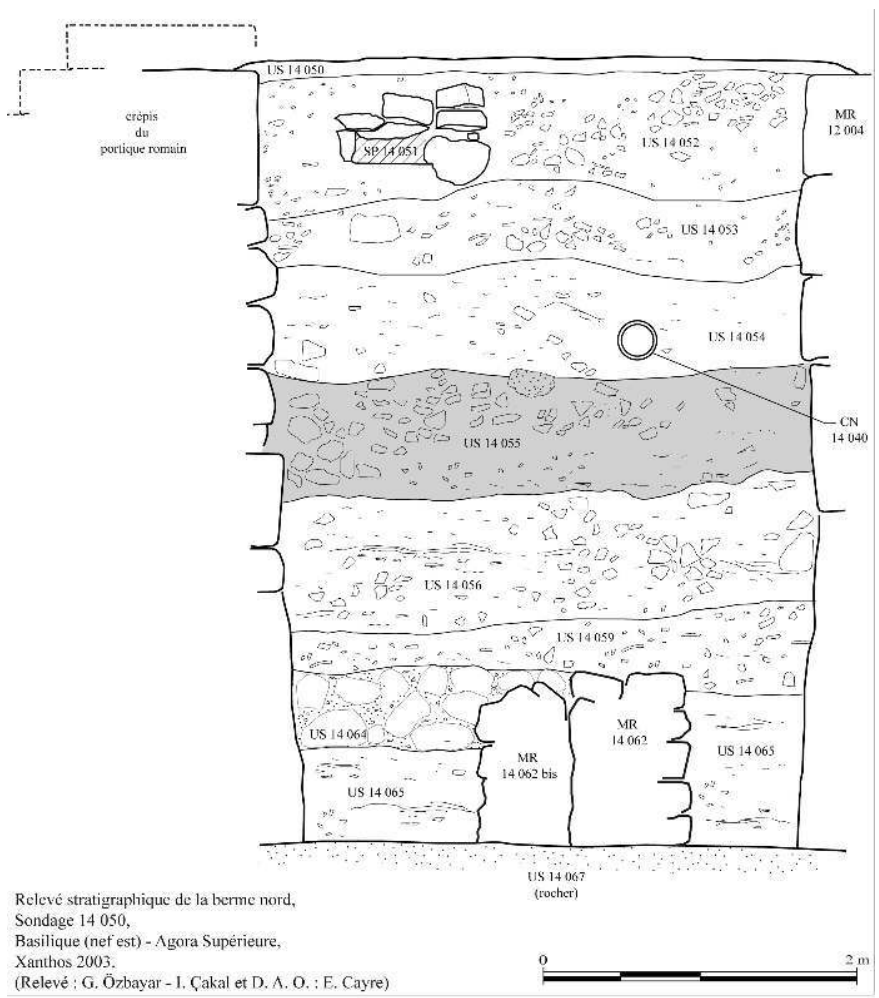

Les découvertes récentes faites à Xanthos complètent la carte des amphores italiques en Lycie. Elles éclairent le fonctionnement d'une économie régionale, dont on commence à percevoir certaines spécificités à la fin de la période hellénistique et à l'époque impériale (Élaigne et Lemaître 2014, p. 588-591).

Située dans l'ouest de la Lycie, en retrait par rapport à la côte et en amont d'une plaine marécageuse correspondant au débouché maritime du fleuve Xanthe, le centre urbain de Xanthos a été exploré de manière relativement intense, en certains points de la ville, par la mission française de Xanthos-Létôon depuis 1951. Les fouilles archéologiques conduites sur le site ont montré une occupation continue entre la fin du viII ou le début du $\mathrm{VII}^{\mathrm{e}}$ siècle, puis au Moyen Âge sous une forme profondément transformée (Des Courtils 2007, p.18-19). Les sources littéraires mentionnent régulièrement la Lycie et Xanthos en particulier, aux époques hellénistique puis impériale. Paradoxalement, les recherches archéologiques sur le site ont montré peu de traces de construction ou de couches d'occupation datées entre le $\mathrm{III}^{\mathrm{e}}$ et le $\mathrm{I}^{\mathrm{er}} \mathrm{s}$. avant notre ère (Des Courtils 2003, p. 4 et Des Courtils 2007, p. 18 et note 27).

De 2001 à 2002, un sondage (Sd 14050), localisé dans la partie méridionale d'un des compartiments de la nef ouest de la basilique civile de l'Agora supérieure de Xanthos, a permis l'exploration des phases de construction du complexe architectural implanté dans ce quartier de la ville (fig. 3$)^{4}$. La fouille du portique a mis au jour une citerne ainsi que des murs en pierre sèche provenant d'un édifice antérieur à la basilique, sans doute d'époque hellénistique (Cavalier 2003, p. 427 et Cavalier 2012, p. 191). Ces structures anciennes, très arasées ont été fossilisées par d'épais apports de terre après la construction de la basilique. Les remblais, mobilisés au moment de l'érection du portique et dont on perçoit bien la succession sur la coupe stratigraphique (fig. 4), ont livré 
beaucoup de mobiliers archéologiques. Parmi eux, une couche (US 14055) a particulièrement retenu notre attention car elle comptait plusieurs centaines de tessons. Malheureusement nous ne disposons d'aucune statistique précise sur l'ensemble du mobilier, comme par exemple la proportion d'amphores par rapport au reste des céramiques.

Les conteneurs commerciaux ont tout de même pu faire l'objet d'une étude plus fine mais qui n'a pas pris en compte l'ensemble des tessons de panses. Seuls les éléments de formes possiblement identifiables ont été examinés de manière détaillée, $i$. e. observation de la pâte afin de déterminer l'origine des tessons et analyse morphométrique notamment pour les conteneurs italiens.

Au total, le remblai a livré 45 fragments correspondant à des bords, des anses et des fonds auxquels il faut ajouter un tesson de panse retaillé pour servir de bouchon (fig. 5). Les éléments se répartissent entre quatre groupes de productions principales, originaires de l'Égée et de Méditerranée occidentale, tandis que neuf fragments d'anses restent sans attribution précise. Rhodes fournit le groupe le plus important avec deux bords, onze anses et deux fonds. Tous les éléments présentent une pâte calcaire de couleur beige à rose, finement épurée et appartiennent à la forme dite «classique » des productions rhodiennes (Finkielsztejn 2001, p. 50). Les deux bords sont en bourrelet relativement mince et légèrement biseauté sur son profil interne (fig. 6, 14055. 9 et 14055. 7). Le second présente un diamètre d'embouchure plus réduit, soit $10 \mathrm{~cm}$ contre $12 \mathrm{~cm}$ pour l'exemplaire précédent. Leur morphologie les rapproche de productions datées du $\mathrm{II}^{\mathrm{e}}$ siècle ou des premières décennies du i ${ }^{\mathrm{er}}$ siècle av. J.-C. (Empereur et Hesnard 1987, p. 61, pl. $3,11,12$ et 13$)$.

Fig. 5 : Xanthos, US 14055. Amphores : éléments de formes par région de production.

\begin{tabular}{|l|l|l|l|l|l|}
\hline Région de production & Type & Bords & Anses & Fonds & Panses \\
\hline \multirow{4}{*}{ Italie } & Dressel 1A & 1 & & & \\
\cline { 2 - 7 } & Dressel 1B & 3 & 4 & & \\
\cline { 2 - 7 } & Dressel 1C & 1 & & & \\
\cline { 2 - 7 } & Dressel 1 & 1 & 2 & 1 & 1 \\
\hline Cnide & & & 4 & & \\
\hline Cos et sa région & Dressel 2/4 & 1 & 2 & 1 & \\
\hline Rhodes & & 2 & 11 & 2 & \\
\hline Indéterminée & & 0 & 9 & & \\
\hline Totaux & & 9 & 32 & 4 & 1 \\
\hline
\end{tabular}


Fig. 6 : Xanthos, US 14055. Amphores d'origine égéenne. Ech. 1/3.

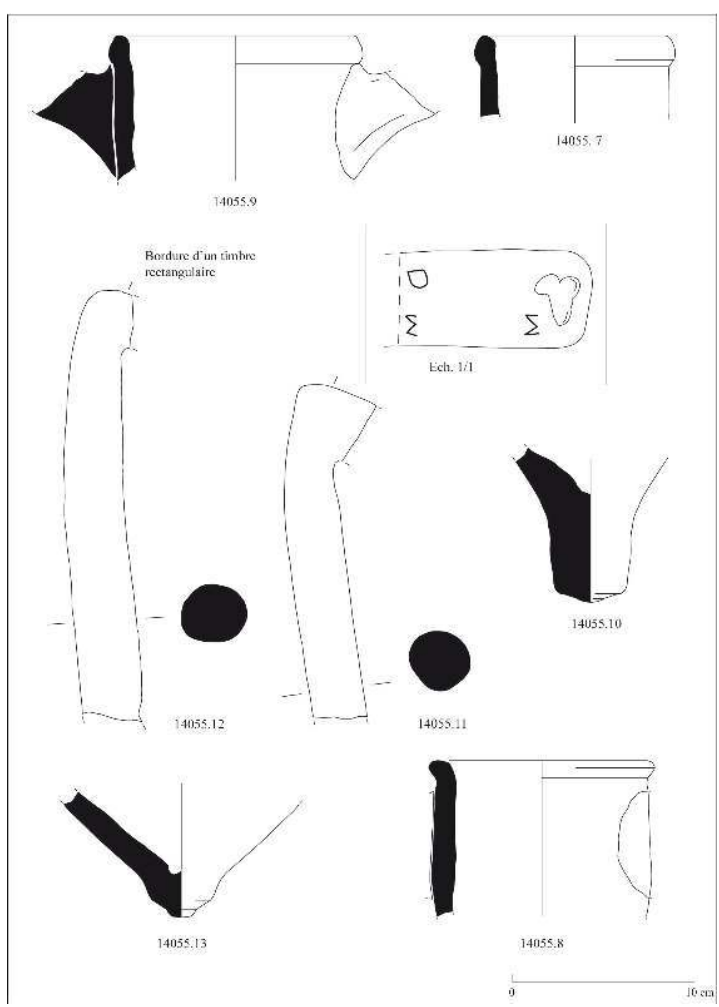

Dessins S. Lemaître.

conserve la trace du bord d'un timbre rectangulaire (fig. 6, 14055. 12). Une autre anse rhodienne porte sur un coude angulaire, relevé cette fois, un timbre rectangulaire aux trois-quarts effacés mais sur lequel apparaît clairement la représentation d'une grappe de raisin dans sa partie droite (fig. 6, 14055.11). Ce timbre peut être rapproché de marques attribuées au fabricant Sotairos, dit «à la grappe de raisin». Elles sont placées dans les petites séries de la période $\mathrm{V}$ du timbrage rhodien, datée de la seconde moitié du $\mathrm{II}^{\mathrm{e}}$ siècle av. J.-C. (Finkielsztejn 2001, p. 154 et tableau 12.2 p. 156, puis p. 166-167). Les autres éléments d'anses de section ronde ne fournissent pas d'informations complémentaires. Un pied cylindrique assez haut s'apparente également plutôt aux productions du $\mathrm{II}^{\mathrm{e}}$ siècle avant J.-C. (fig. 6, 14055. 10).

Le répertoire des importations égéennes est complété par quatre éléments appartenant à des amphores à anses bifides, dont la cité de Cos a sans doute fourni le modèle (Grace 1979, ill. 56 et texte page précédente). Le petit pied court «à bouton rentré » (fig. 6, 14055. 13) est caractéristique des productions de Cos et de sa région au $\mathrm{III}^{\mathrm{e}}$ ou $\mathrm{II}^{\mathrm{e}}$ siècle avant J.-C. avant d'évoluer vers une pointe évasée, puis un pied cylindrique (Empereur et Hesnard 1987, p. 22 et pl. 4, 19). Enfin, un fragment de col à bord en bourrelet fin sur lequel on peut voir la trace de l'attache de l'anse correspond également à ces productions. La pâte de ces deux fragments est fine, de couleur rose foncé et riche en paillettes de mica, associée à une surface de couleur beige qui renvoie plutôt aux productions de même forme qui se multiplient en dehors de l'île de Cos à partir de la fin du $\mathrm{II}^{\mathrm{e}}$ siècle et aussi au $\mathrm{I}$ er siècle avant notre ère, dans la région proche sur le continent (Empereur et Hesnard 1987, p. 23 ; Empereur et Picon 1989, p. 225-226). Deux anses bifides doivent être associées 
à ce groupe de production. Elles se caractérisent par leur gracilité ( $44 \mathrm{~mm}$ de large et $23 \mathrm{~mm}$ d'épaisseur pour l'une et $40 \mathrm{~mm}$ de large et $20 \mathrm{~mm}$ d'épaisseur pour l'autre).

Un dernier groupe d'amphores égéennes complète le corpus des conteneurs grecs. Il est constitué par quatre fragments d'anses appartenant à des récipients cnidiens non illustrés. De section ovale aplatie, les tessons ont une pâte caractéristique, brun rouge, riche en dégraissants de nature et dimensions variées, tantôt gris, tantôt blancs de forme anguleuse mesurant jusqu'à $2 \mathrm{~mm}$ de diamètre.

Fig. 7 : Xanthos, US 14055. Amphores italiques les mieux conservées.

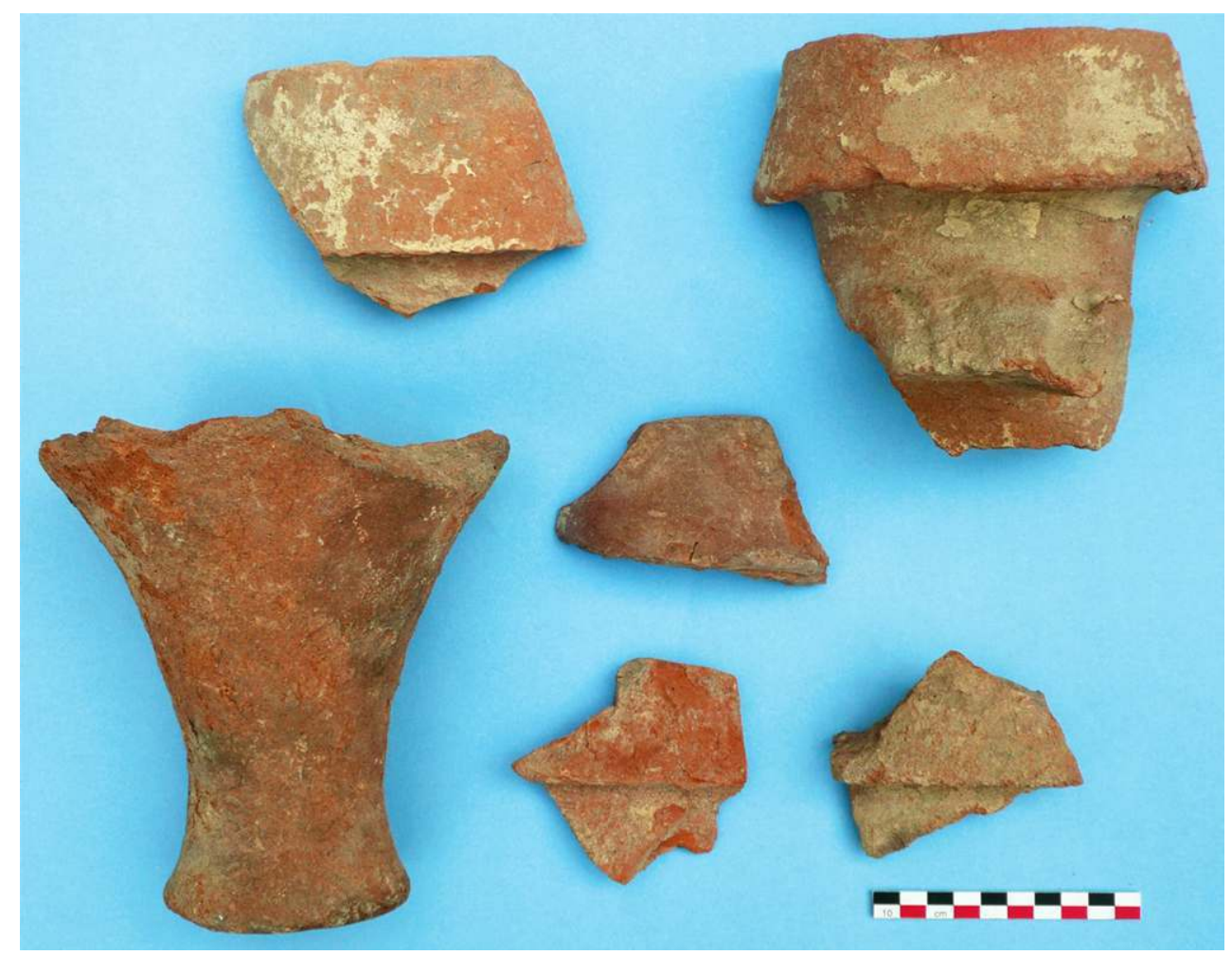

Cliché S. Lemaître.

Dans ce lot, les importations occidentales rassemblent quatorze éléments appartenant à des amphores de la famille des Dressel 1 originaires de la façade tyrrhénienne de la péninsule italienne (fig. 5). Les éléments sont fragmentaires (fig. 7).

\section{Catalogue des amphores italiques}


Fig. 8 : Xanthos, US 14055. Amphores d'origine italique. Ech. 1/3.

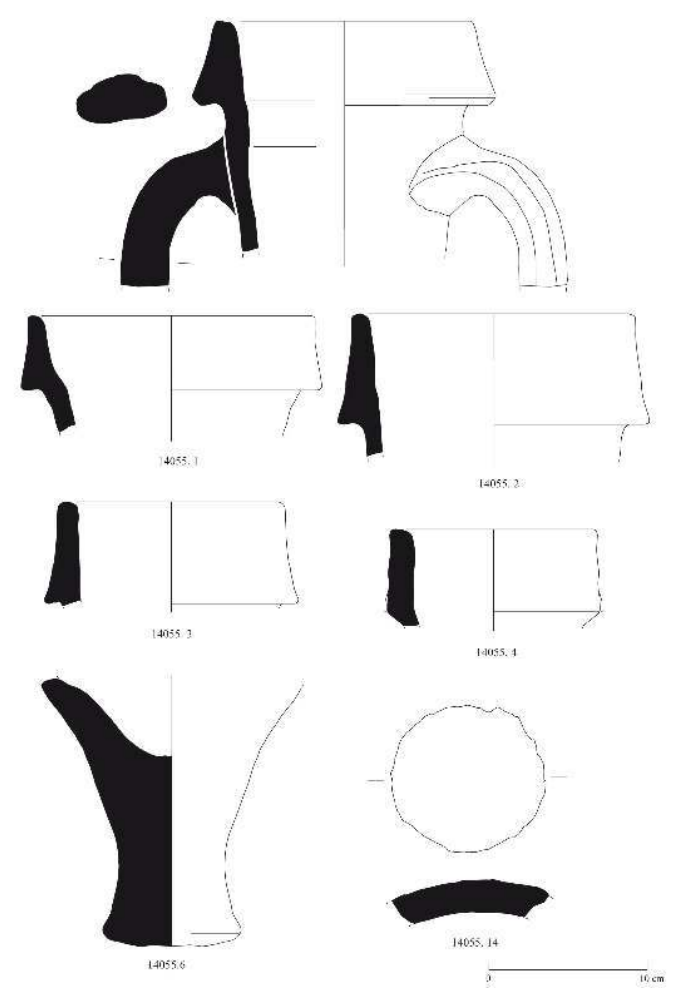

Dessins S. Lemaître.

-14055. 1 (fig. 8): Bord d'amphore Dressel 1A à lèvre en bandeau. Hauteur: $47 \mathrm{~mm}$. Largeur : $28 \mathrm{~mm}$. Diamètre d'embouchure : $160 \mathrm{~mm}$. Inclinaison de la lèvre : $85^{\circ}$. Classe 2 ou 3. Pâte de couleur rouge, contenant des inclusions blanches et grises. Surface érodée.

-14055. 2 (fig. 8) : Bord d'amphore Dressel 1B à lèvre en bandeau haut. Hauteur : 70 mm. Largeur : $27 \mathrm{~mm}$. Diamètre d'embouchure : $170 \mathrm{~mm}$. Inclinaison de la lèvre : $81^{\circ}$. Classe 3. Pâte de couleur brun rose contenant des inclusions fines noires (d'origine volcanique ?) peu abondantes. Surface beige.

$43 \quad$-14055. 3 (fig. 8): Bord d'amphore Dressel $1 \mathrm{C}$ à lèvre en bandeau haut et incliné vers l'intérieur. Hauteur : $65 \mathrm{~mm}$. Largeur : $23 \mathrm{~mm}$. Diamètre d'embouchure : $140 \mathrm{~mm}$. Inclinaison de la lèvre: $81^{\circ}$. Classe 3. Pâte de couleur brun orangé contenant quelques rares inclusions blanches et des nodules gris de nature indéterminée.

-14055. 4 (fig. 8) : Bord d'amphore Dressel $1 \mathrm{C}$ à lèvre en bandeau haut et incliné vers l'intérieur. Hauteur : $54 \mathrm{~mm}$. Largeur : $17 \mathrm{~mm}$ minimum conservés. La partie inférieure de la lèvre est fragmentaire. Diamètre d'embouchure : $130 \mathrm{~mm}$. Inclinaison de la lèvre : $83^{\circ}$. Classe 3. Pâte de couleur rouge sableuse contenant de très nombreuses inclusions fines noires et brillantes, vraisemblablement d'origine campanienne (Pompéi ?).

-14055. 5 (fig. 8) : Bord et anse d'amphore Dressel 1A à lèvre haute de section triangulaire. Hauteur : $54 \mathrm{~mm}$. Largeur : $32 \mathrm{~mm}$. Diamètre d'embouchure : $160 \mathrm{~mm}$. Inclinaison de la lèvre: $73^{\circ}$. Classe 2 ou 3. L'anse de section aplatie est marquée par une large nervure centrale. Largeur de l'anse : $56 \mathrm{~mm}$. Épaisseur de l'anse : $29 \mathrm{~mm}$. Pâte de couleur rouge sableuse contenant de très nombreuses inclusions fines noires et brillantes, vraisemblablement d'origine campanienne (Pompéi ?). Surface de couleur crème. 

Hauteur : $120 \mathrm{~mm}$. Diamètre : $86 \mathrm{~mm}$. Pâte de couleur rouge très riche en inclusions fines noires et brillantes, vraisemblablement d'origine campanienne (Pompéi ?). La surface est érodée.

14055. 14 (fig. 8) : Panse retaillée probablement pour servir de bouchon. Diamètre $95 \mathrm{~mm}$ x $90 \mathrm{~mm}$. Épaisseur : $20 \mathrm{~mm}$. Pâte de couleur brun rose très riche en inclusions noires et brillantes, vraisemblablement d'origine campanienne (Pompéi ?). Surface de couleur crème.

-14055. 15 : Anse d'amphore Dressel 1 de section ovale aplatie marquée par une nervure centrale (14 mm de large). Largeur : $51 \mathrm{~mm}$. Épaisseur : $32 \mathrm{~mm}$. Pâte de couleur rouge contenant des nodules gris ainsi que quelques inclusions blanches de nature indéterminée.

-14055. 16 : Anse d'amphore Dressel 1 de section ovale marquée par avec nervure centrale très nette. Largeur : $51 \mathrm{~mm}$. Épaisseur : $28 \mathrm{~mm}$. Pâte de couleur rouge très riche en inclusions fines noires et brillantes, vraisemblablement d'origine campanienne (Pompéi ? ).

50 -14055. 17 : Anse d'amphore Dressel 1 (?) de section ovale. Largeur : $43 \mathrm{~mm}$. Épaisseur : $28 \mathrm{~mm}$. Pâte de couleur beige contenant des inclusions rouges et grises.

51 -14055. 18: Anse d'amphore Dressel 1 de section ovale. Largeur: $46 \mathrm{~mm}$. Épaisseur: $34 \mathrm{~mm}$. Pâte de couleur rouge très riche en inclusions fines noires et brillantes, vraisemblablement d'origine campanienne (Pompéi ?).

Les amphores italiques Dressel 1 ont fait l'objet de prise de mesures spécifiques (fig. 9) ${ }^{5}$ La hauteur, la largeur, l'épaisseur, l'inclinaison et le diamètre ont été mesurés sur les bords, la hauteur et le diamètre sur le fond et la largeur et l'épaisseur sur les anses, lorsque l'état de conservation le permettait. Certaines de ces mesures ont ensuite été associées afin de produire une représentation graphique du lot d'amphores. Pour cette étude, nous avons choisi la combinaison Hauteur/Inclinaison des lèvres qui conduit à définir, pour les individus représentés par un bord, leur appartenance à une classe coïncidant avec une période de production (Guichard 1997, p. 134-135).

Fig. 9 : Caractéristiques morpho-métriques des amphores italiques Dressel 1 découvertes dans l'US 14055.

\begin{tabular}{|l|l|l|l|l|l|l|l|}
\hline Inventaire & Frgt & H & L & Dia & Incl & Classe & Type \\
\hline 1 & bord & 47 & 28 & 170 & 85 & 2 ou 3 & D1A tardive \\
\hline 2 & bord & 70 & 27 & 170 & 81 & 3 & D1B \\
\hline 3 & bord & 65 & 23 & 140 & 81 & 3 & D1C \\
\hline 4 & bord & 54 & 17 min. & 130 & 83 & 3 & D1C \\
\hline 5 & bord & 54 & 32 & 160 & 73 & 2 & D1A tardive \\
\hline 6 & fond & 120 &. & 86 &. &. & D1B \\
\hline
\end{tabular}

53 V. Guichard a déterminé trois classes principales et deux classes intermédiaires caractérisées de la manière suivante : 
54 - Classe 1 (Gréco-italique)

Hauteur = jusqu'à $35 \mathrm{~mm}$

Inclinaison $=$ jusqu'à $60^{\circ}$

55 - Classe 1 ou 2 (Gréco-italique/Dressel 1A de transition)

Hauteur $=30$ à $35 \mathrm{~mm}$

Inclinaison $=50$ à $70^{\circ}$

56 - Classe 2 (Dressel 1A)

Hauteur $=30$ à $60 \mathrm{~mm}$

Inclinaison $=50$ à $95^{\circ}$

57 - Classe 2 ou 3 (Dressel 1A tardive)

Hauteur $=45$ à $60 \mathrm{~mm}$

Inclinaison $=75$ à $95^{\circ}$

58 - Classe 3 (Dressel 1B)

Hauteur $=$ de $45 \mathrm{~mm}$ à plus de $70 \mathrm{~mm}$

Inclinaison $=75$ à $110^{\circ}$

59 Ce type d'analyse permet de définir, au sein de contextes archéologiques fiables et à partir d'un ensemble de conteneurs, un faciès morphologique et chronologique des arrivages d'amphores italiques d'époque républicaine. Il est largement utilisé aujourd'hui sur les sites laténiens de Gaule (Guichard 1997, Maza 1998, Loughton 2000, Benquet, Piot 2000, Poux 2004, Loughton, Alberghi 2012). Ses attraits et ses limites ont été mises en lumière depuis longtemps (Maza 1998) et plus récemment (Olmer 2012), mais cette approche reste pertinente à nos yeux lorsqu'elle associe à l'étude des bords, celle des anses et des fonds d'amphores.

60 La hauteur des lèvres varie entre $47 \mathrm{~mm}$ et $70 \mathrm{~mm}$ pour une moyenne établie à $58 \mathrm{~mm}$, soit au-dessus du seuil déterminé entre Dressel 1A et Dressel 1B (Tchernia 1986a ou 1986b, p. 313). Les diamètres d'embouchure, compris entre 130 et $170 \mathrm{~mm}$, sont plutôt faibles.

61 Dans le lot xanthien, les mesures des bords et la combinaison de la hauteur et de l'inclinaison de la lèvre permettent de déterminer deux individus dans la classe 2 ou 3, de transition entre les variantes Dressel 1A et Dressel 1B (fig.8, 14055.1 et 5) et un exemplaire qui intègre la classe 3, soit une amphore Dressel 1B (fig. 8, 14055. 2). Enfin, deux conteneurs (fig. $8,14055.3$ et 4), à faible diamètre d'embouchure, respectivement 140 et $130 \mathrm{~mm}$, à lèvre haute et profil rentrant, doivent être rapprochés de la variante Dressel 1C (Tchernia 1986, p. 320), dont la production se place traditionnellement dans le dernier quart $\mathrm{du} \mathrm{II}^{\mathrm{e}}$ siècle et dans les premières décennies du $\mathrm{I}^{\mathrm{er}}$ siècle $\mathrm{av}$. J.-C. (Poux et Selles 1998, p. 212 ; la datation proposée est un peu plus large dans Empereur et Hesnard 1987, p. 31-32 en dernier lieu Olmer 2012, p. 323).

62 Le fond massif et haut (fig. 8, 14055.6) appartient à une amphore de type Dressel 1A tardive (d'après Stöckli dans Tchernia 1986a ou 1986b, p. 311). Les anses, assez épaisses et larges se rattachent plutôt à la variante tardive de l'amphore Dressel 1. Les conteneurs italiques montrent une relative homogénéité dans la mesure où ils se présentent sous la forme de conteneurs à lèvre en bandeau haut, voire très haut, mais ils correspondent aux trois variantes du type Dressel $1 \mathrm{~A}, 1 \mathrm{~B}$ et $1 \mathrm{C}$ produites à la fin du $\mathrm{II}^{\mathrm{e}}$ siècle et dans le siècle suivant. Les anses associées à l'ensemble ont des largeurs comprises entre 43 et $51 \mathrm{~mm}$, ce qui renvoie aux dimensions observées sur des amphores Dressel 1A tardives (Stöckli 1979, p. 137). 
63 conduisent à donner les dernières décennies du $\mathrm{II}^{\mathrm{e}}$ siècle et peut-être le milieu du siècle
suivant comme fourchette de datation pour la production des conteneurs italiques
retrouvés à Xanthos. Toutes les amphores identifiées dans le remblai 14055 étaient
semble-t-il destinées au transport et à la commercialisation du vin ${ }^{6}$. Leur étude
typologique montre un ensemble de conteneurs égéens et italiques que l'on peut dater
entre le milieu du $\mathrm{II}^{\mathrm{e}}$ siècle et le milieu du dernier siècle avant notre ère.

Fig. 10 : Xanthos, US 14055. Céramiques à parois fines italiques.

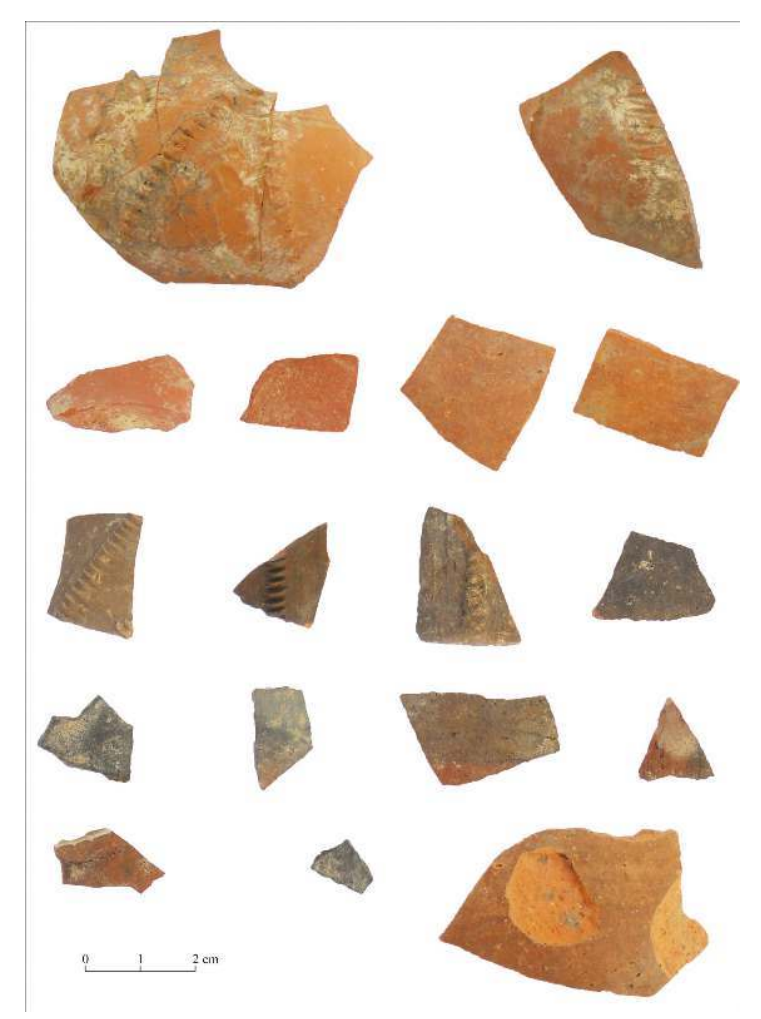

Cliché S. Lemaître. 
En revanche, nous n'avons pas eu l'occasion d'examiner la composition de l'assemblage des céramiques communes afin de conforter la chronologie de l'ensemble et pour y détecter éventuellement des importations italiques, attestées ailleurs en Lycie, sur le site du Létôon par exemple. Le fond d'un plat à cuire à vernis rouge pompéien était associé à un dépotoir mis au jour dans la partie nord dans le sanctuaire (carrés de fouille NE/D-E 17)9. Toutefois, les études menées en Lycie sur d'autres ensembles de mobiliers du HautEmpire et de l'antiquité tardive ont bien montré que le faciès des assemblages des céramiques culinaires était marqué par la faiblesse des importations extérieures à la région. Ces dernières sont essentiellement égéennes, peut-être d'Éphèse, et levantines (voir en dernier lieu Lemaître et al. 2013). Cela ne signifie pas que les importations de céramiques communes italiques n'aient pas existé aux époques précédentes mais simplement qu'elles ne sont peut-être pas encore documentées car extrêmement limitées.

Au final, l'analyse faite sur le mobilier amphorique du remblai 14055 montre la présence de plusieurs conteneurs à vin tyrrhéniens, associés à d'autres amphores égéennes de Rhodes, Cos et Cnide, très largement commercialisés dans le bassin oriental de la Méditerranée. La faiblesse numérique de l'ensemble limite nécessairement la portée des interprétations en terme d'économie.

Le tableau des amphores italiques découvertes en Lycie peut être complété par d'autres éléments provenant de deux sites proches de Xanthos : le sanctuaire du Létôon et Patara.

\section{- Indices dans le sanctuaire du Létôon}

Fig. 11 : Létôon de Xanthos. Amphores italiques Dressel 1.

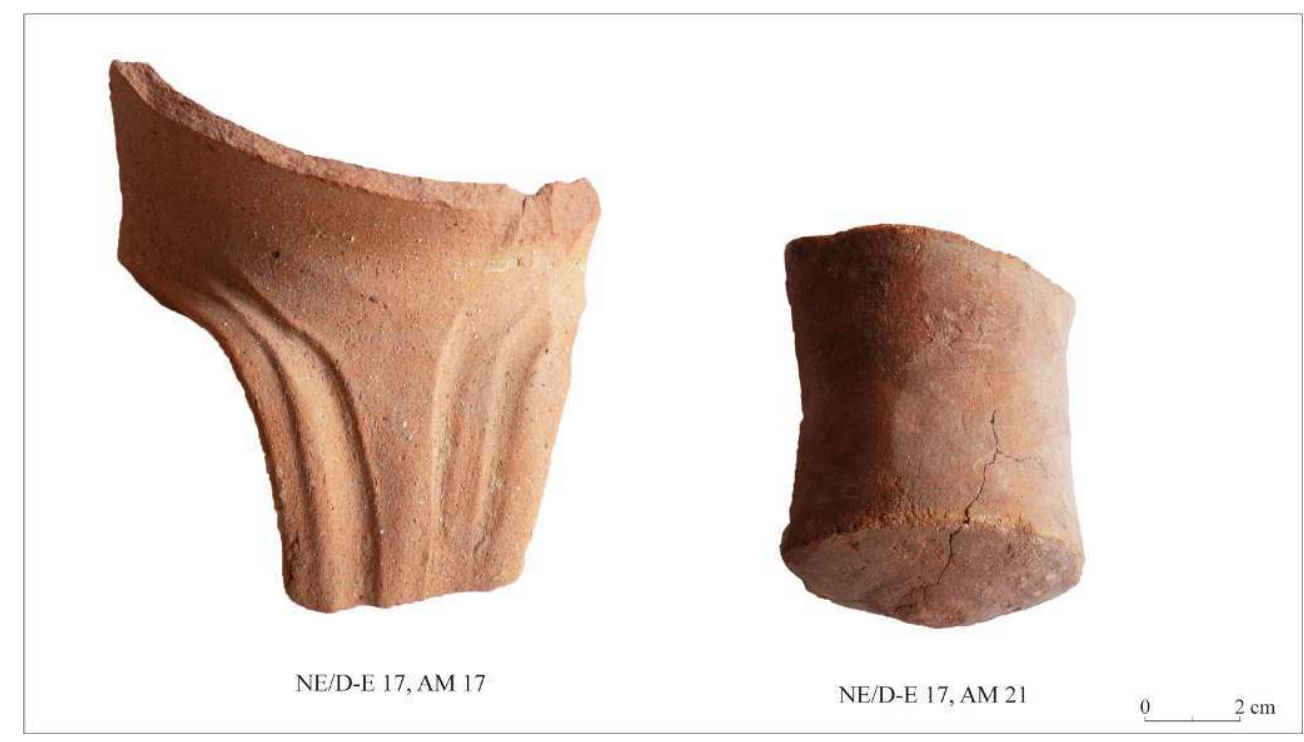

Clichés S. Lemaître.

En 1972, l'exploration par H. Metzger de la zone nord du sanctuaire du Létôon a mis au jour un important remblai auquel étaient associées de grandes quantités de mobiliers en verre, en céramiques ainsi que des restes de faune (Lemaître, Élaigne et Alary 2004) ${ }^{10}$. Les importations italiques y sont matérialisées par près d'une trentaine d'éléments de formes (bord, fonds, anses et col) rattachés à plusieurs groupes de production d'époque républicaine et impériale. Neuf fragments appartiennent à des amphores Dressel 1, 
variantes $1 \mathrm{~B}$ et $1 \mathrm{C}$ (fig. 11), trois anses et deux fonds correspondent à des conteneurs de type Lamboglia 2, produits sur la façade adriatique. Les arrivages italiques d'époque impériale sont représentés dans le remblai par douze fragments (fonds, anses et col) rattachés à des amphores à vin Dressel 2/4 ainsi qu'un bord et deux fonds de conteneur Dressel 21/22 à sauces et conserves de poisson.

\section{- Timbres sur amphores à Patara}

Fig. 12 : Amphores italiques de Patara.
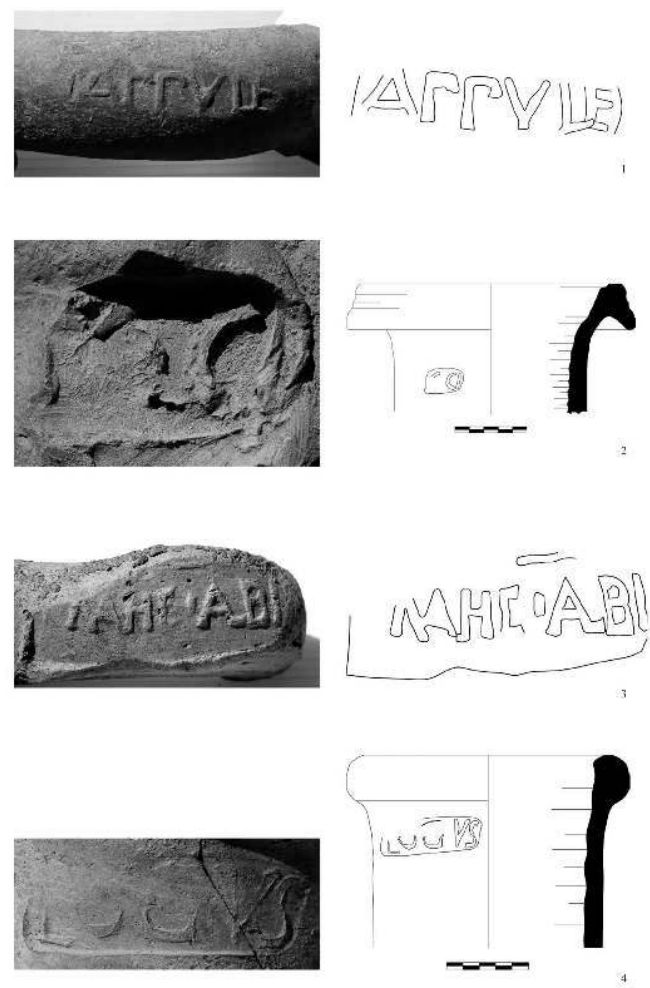

D’après Dündar 2013, p. 149.

À Patara, la publication récente d'un article d'E. Dündar consacré aux timbres sur amphores italiques mises au jour sur le site ${ }^{11}$ rend désormais visibles les conteneurs, dont on pouvait supposer l'existence, étant donné la place et le statut important qu'occupait la cité portuaire de Patara aux époques hellénistique et romaine en Lycie (Dündar 2013). Plus précisément, l'étude porte sur quatre timbres sur amphore, découverts en différents points de la ville mais sans contexte archéologique précisément défini et dont nous reproduisons l'illustration ici. Le premier appartient à une amphore à huile de Brindes (fig. 12, 1), un type non encore attesté en Lycie jusqu'à présent. Le deuxième timbre (fig. 12, 2) est porté sur le col d'une amphore Dressel 1A (Tchernia 1986, p. 316-317), plutôt que d'une Dressel 1B (Dündar 2013, p. 145, fig. 2b). En effet, le bord de section courte triangulaire, associé à un diamètre d'embouchure égal à $160 \mathrm{~mm}$ correspond à la morphologie d'un groupe d'amphores Dressel 1A tardives. Deux autres timbres, l'un sur l'anse (fig. 12, 3) et le second sur un col (fig. 12, 4), apparaissent sur des amphores italiques Dressel 2/4. La description de la pâte faite pour le premier timbre, lu MAHE (. ALBI (, ne permet pas de préciser l'origine du fragment (Dündar 2013, p. 145). Aux 
rapprochements proposés par l'auteur, nous pouvons ajouter que le nom abrégé MAHE pour Mahes ou Mahetis se retrouve sur des estampilles sur amphores de l'atelier d'Albinia dans l'ager Cosanus qui a produit des amphores gréco-italiques, Dressel 1 et Dressel 2/4 (Olmer 2003, p. 201). Il apparaît également sur le timbre du dolium n ${ }^{\circ} 14$ associé à la cargaison de l'épave varoise Grand Ribaud D qu'A. Hesnard relie au timbre du dolium n ${ }^{\circ} 13$ de la même cargaison, signé.HELVIVS/MAHESFE (Hesnard et al. 1988, p. 41 pour le dolium $\mathrm{n}^{\circ} 14$ et $\mathrm{p} .40$ pour le dolium n ${ }^{\circ} 13$ ). L'analyse de l'épave et de sa cargaison, composée de dolia estampillés, pour certains originaires de Minturnes, et d'amphores de type Dressel 2/4, d'origine pompéienne pour la majorité d'entre elles, conduit les auteurs à replacer le point de départ du navire dans un port de Campanie, où les dolia ont été remplis et les amphores chargées à bord (Hesnard et al. 1988, p. 144). Le quatrième timbre découvert à Patara sur amphore Dressel 2/4, assez mal conservé (fig. 12, 4), est lu LCCVS (Dündar 2013, p. 146). À ces quatre exemplaires il faut sans doute ajouter un certain nombre d'amphores italiques non timbrées qui viendront à n'en pas douter étoffer le corpus des importations italiques en Lycie.

70 Le tableau des découvertes d'amphores italiques dans cette région appellent quelques commentaires. À l'heure actuelle, il semble que ces conteneurs se concentrent dans les sites urbains en zone littorale ou proche du littoral d'une part: Xanthos, Patara et dans le sanctuaire fédéral des Lyciens : le Létôon, d'autre part. Nous ne savons rien des amphores peut-être mises au jour à Caunos de Carie, un port important à la frontière occidentale de la Lycie ou encore à Andriakè, un port de Lycie orientale. Une série de prospections menées par une équipe autrichienne en différents points de la ville et notamment près des horrea en 2008 n'a pas révélé d'indices d'importations italiques ${ }^{12}$. L'étude des mobiliers livrés par les fouilles, réalisées depuis par N. Çevik, apportera peut-être des informations sur la présence des conteneurs italiques dans le site portuaire même si l'essentiel des découvertes concerne l'antiquité tardive (Çevik, Bulut et Akyürek 2012, p. 71).

71 Un peu plus à l'est, dans la même zone, les conteneurs italiques n'ont pas été repérés à Limyra et elles ne sont pas, à notre connaissance, signalées non plus dans l'Hinterland lycien.

Cependant, cette répartition doit sans doute plus à la géographie des explorations archéologiques et au manque de publications ou même des études sur les amphores qu'à la réalité des arrivages antiques. L'arrière pays lycien, marqué par une forte densité d'occupation, notamment à l'époque classique, voit un grand nombre de sites abandonnés au-delà du III ${ }^{\mathrm{e}}$ siècle av. J.-C. au profit de sites plus proches du littoral (des Courtils 2003).

L'observation des pâtes argileuses, avec lesquelles les amphores mises au jour à Xanthos ont été façonnées, montre qu'une partie des conteneurs italiques républicains découverts en Lycie sont des productions de la côte tyrrhénienne. À l'intérieur de ce groupe comprenant vraisemblablement des conteneurs d'Étrurie, les amphores originaires de Campanie se distinguent nettement. La présence des productions campaniennes, sous la forme d'amphores Dressel 1A tardives, Dressel 1B et Dressel 1C, semble s'accentuer lorsqu'on prend en compte les amphores Dressel $2 / 4$ à partir du milieu du $\mathrm{I}^{\mathrm{er}}$ siècle avant J.-C. et encore au siècle suivant. À cette période-là, les amphores identifiées comme italiques (et campaniennes) ont une pâte rouge foncé, très riche en minéraux noirs brillants sans doute d'origine volcanique ${ }^{13}$. 

l'estuaire du fleuve Xanthe a pu également servir de lieu de mouillage (des Courtils 2007, p.11-12). P. Arnaud résume ainsi le point stratégique que constituait la Lycie dans les routes commerciales du bassin oriental de la Méditerranée: «[...] Patara et Myra pouvaient sans mal échanger directement avec l'Égypte et se trouvaient sur les lignes de Chypre et du Levant dans les deux sens et, nécessairement, sur la route d'Égypte à Rhodes » (Arnaud 2011, p. 415). Les relations entre la Lycie et l'Égypte au cours de la période hellénistique ont été maintes fois soulignées, en grande partie à cause de la tutelle exercée par les Lagides sur la région durant le $\mathrm{III}^{\mathrm{e}}$ siècle (parmi d'autres Robert 2/1978, p. 279, des Courtils 2007, p. 17).

D’après P. Arnaud, les lieux lyciens mentionnés dans les sources antiques, le Stadiasme de la Grande Mer en particulier, sont mis en relation avec deux niveaux des circulations maritimes : les ports principaux et les mouillages remarquables comme points de départ ou d'arrivée de navires engagés dans des itinéraires directs vers Rhodes ou vers l'Égypte par exemple, aux côtés de toponymes liés à des segments de navigation courts, proches de la côte et fréquentés dans le cadre d'échanges locaux ou régionaux (Arnaud 2011, p. 430). 


\section{- Routes suivies par les amphores italiques arrivées en Lycie}

80 À cause des limites ou des contraintes techniques des navires antiques, on comprend aisément que les bateaux transportant le vin italique aient également emprunté les routes déterminées par les courants marins et le régime des vents. Un examen des données publiées montrent que les amphores italiques d'époque républicaine puis impériale sont régulièrement attestées, à raison de moins d'une dizaine d'exemplaires, le long de la côte sud-anatolienne, plus à l'est en Cilicie. En témoignent les amphores découvertes en mer qui composent les collections de trois musées de la région. Ainsi, parmi les conteneurs exposés du musée d'Anamur, on identifie deux amphores Lamboglia 2 et Dressel $6 \mathrm{~A}^{14}$. Une amphore gréco-italique (şenol 2009, p. 215), deux amphores Lamboglia 2 (şenol 2009, p. 216-217) et trois amphores Dressel 2/4 (şenol 2009, p. 218-219) provenant du musée de Taşucu ont aussi été publiées ${ }^{15}$. À Silifke, le musée conserve au moins une amphore de Brindes, tandis qu'une amphore Dressel 2/4 italique est conservée à Elaiussa Sébaste (Ferrazzoli et Ricci 2009, p. 42, fig. 4). Elles apparaissent ensuite dans les sites antiques de la côte levantine comme Beyrouth (Lemaître 2007, p. 280-281) et bien sûr Alexandrie ${ }^{16}$.

81 Une arrivée par l'Ouest des produits italiques en Lycie peut être supposée étant donné l'origine occidentale des amphores à vin tyrrhéniennes. Le passage de ces bateaux serait intervenu plutôt en saison estivale si on suit les sens de navigation principaux dictés par les conditions naturelles. On ne peut cependant pas complètement exclure la possibilité d'une redistribution des produits italiques depuis l'Égypte, en suivant la côte de port en port, vers l'est, le nord et finalement vers l'ouest. Mais ce trajet allongerait considérablement les temps d'acheminement des marchandises.

Les découvertes d'amphores Dressel 2/4 italiques en Galatie, montrent également que les marchandises occidentales pénétraient le coeur de l'Anatolie à l'époque impériale, mais pas nécessairement par la côte sud-anatolienne (Monsieur et De Paepe 2002).

$\mathrm{Au}$ croisement de deux routes maritimes importantes, d'ailleurs signalées par les géographes antiques, le relais rhodien a sans doute joué un rôle déterminant dans l'acheminement des denrées italiques vers le sud de l'Anatolie. Les conteneurs italiens de type gréco-italique (Will type 1d) et Lamboglia 2 sont attestés à Rhodes même (Lund 2000, p. 81 et 85). Par ailleurs Rhodes a entretenu avec la Lycie des relations particulières et changeantes depuis le $\mathrm{IV}^{\mathrm{e}}$ siècle avant notre ère (Bresson 1999). Les territoires de la Pérée rhodienne s'étendaient jusqu'à la petite île de Mégisté située en face d'Antiphellos à la frontière orientale de la Lycie (Bresson ibid.). Pendant une vingtaine d'années, à partir de 188 av. J.-C., les Rhodiens obtinrent même de Rome le contrôle de la Lycie jusqu'à ce que les Lyciens réclament auprès du Sénat romain d'être libérés de la tutelle rhodienne, ce qui fut fait en 167 av. J.-C. (Bresson 1999, p. 104-106). Parallèlement, la présence à Rhodes et à Caunos, situé en Carie, à la frontière occidentale de la Lycie, d'Italiens et de Romains est bien attestée et la formation d'un conventus de negotiatores dès le $\mathrm{I}^{\mathrm{er}}$ siècle av. J.-C. est sérieusement envisagé (Bresson 2002).

Plus à l'ouest la côte sud de la Crète constituait aussi une étape importante dans l'acheminement des vins italiques par deux routes principales, soit en remontant vers la côte occidentale de la Grèce et le Péloponnèse pour une traversée de l'Adriatique, vers Brindes notamment, soit en navigation hauturière vers le sud de la Sicile puis le détroit de Messine pour rejoindre les villes d'Italie du sud sur la façade tyrrhénienne (Arnaud 
2005, p. 175 et 178-179). Il est bien établi que la Campanie et Pouzzoles en particulier entretenaient des liens privilégiés avec la Méditerranée orientale, notamment Alexandrie, pour le commerce des esclaves, des marbres et des oeuvres d'art (Tchernia 1986, p. 73). Pour P. Arnaud les routes «directes » d'ouest en est les plus normalement fréquentées en Méditerranée centrale, celles qui concernent vraisemblablement l'acheminement des amphores italiques vers l'est, utilisaient la Sicile comme «point d'articulation » (Arnaud 2005, p. 180).

\section{- Contexte politique et économique : Délos et les amphores Lamboglia 2} Xanthos, correspond à une période charnière dans l'organisation des échanges commerciaux entre l'Italie et le bassin oriental de la Méditerranée à savoir les années comprises entre 150 et 50 environ avant notre ère. À ce moment-là, la Confédération lycienne est affranchie de la tutelle rhodienne. Elle organise un concours sacré en l'honneur de la déesse Rome vers le milieu du II ${ }^{\mathrm{e}}$ siècle av. J.-C. (Robert 1978, p. 289) dont les épreuves thyméliques se déroulent sans doute dans le théâtre du Létôon (Badie, Lemaître et Moretti 2004, p. 145). La mise en place de ce concours est interprétée comme un signe de la volonté des Lyciens de montrer leur fidélité envers Rome (des Courtils 2007, p. 19).

Méditerranée orientale à la basse époque hellénistique mettent en lien la montée en puissance de l'emporion délien et la baisse de l'économie rhodienne. Le changement interviendrait lors de l'obtention par Délos du statut de port franc à partir de 167 avant notre ère. Les marchands y arriveraient plutôt après la destruction de Corinthe en 146 (Etienne 2002, p. 5). La plupart des marchands italiens semblent avoir quitté l'île après 88 et 69 av. J.-C., même si l'on sait aujourd'hui que Délos continue à être fréquentée après cette date (Etienne 2002, p. 7). Ce schéma ferait de Délos un passage obligé des bateaux chargés d'amphores italiques destinée à être commercialisées en Méditerranée orientale.

Il convient de s'interroger sur le caractère obligatoire de l'étape délienne pour les bateaux qui ont acheminé les amphores italiques sur la côte sud-anatolienne.

À Délos, les amphores italiques sont très nombreuses, plusieurs centaines d'individus sont mentionnés (Empereur 1983, p. 97-98). Il s'agit essentiellement d'amphores de Brindes, de conteneurs de type Lamboglia 2 originaires de la façade adriatique de la péninsule italienne, mais également par ceux produits sur la façade tyrrhénienne, en quantités beaucoup moins importantes (Empereur 1983 et 1986). La communauté des Italiens, notamment du sud de l'Italie était particulièrement bien représentée à Délos (Hatzfeld 1919; Couilloud 1974, p.331; Müller et Hasenohr 2002). Aujourd'hui une opinion communément admise est que les amphores mises au jour sur place correspondent plutôt aux vestiges de consommations des populations italiques vivant dans l'île et souhaitant peut-être continuer à boire les vins auxquels elles étaient accoutumées (Etienne 2002, p. 6). Plus largement, les restes d'amphores présents à Délos sont le reflet de consommations d'une population cosmopolite. Les vins italiens y côtoyaient d'autres vins grecs dont on sait que les élites romaines en Italie étaient accoutumées à les boire (Tchernia 1986, p. 100-106).

Cahiers « Mondes anciens », 7 | 2015 
89 La présence massive des amphores Lamboglia 2 ailleurs en Égée semble étayer l'hypothèse de l'importance de ce que $\mathrm{M}$. Lawall a appelée la « zone Adriatique » évoquée précédemment, au sein de laquelle Délos aurait peut-être servi de relais. Ainsi à Corinthe, des assemblages datés $\mathrm{du} \mathrm{II}^{\mathrm{e}}$ siècle présentent un faciès dans lequel ces amphores dépassent en nombre les conteneurs égéens (Lawall 2004, p. 272). L'examen des rares épaves en mer Égée dont la cargaison comprenait des amphores italiques, révèle la présence exclusive d'amphores Lamboglia 2 de l'Adriatique. Mis à part les exemples mentionnés par M. Lawall à Preveza près d'Actium, à Siphnos ou encore à Thasos (Lawall 2004, p. 273), il faut ajouter l'épave de Kizılburun localisée près de Claros en Turquie. Les restes du navire fouillés entre 2005 et 2011 transportait une cargaison de marbre du Proconnèse destinée au chantier du temple d'Apollon dans le sanctuaire de Claros et en complément, deux douzaines d'amphores dont huit de type Lamboglia 2 associées à un peu plus d'une dizaine d'amphores égéennes datées $d u$ i ${ }^{\mathrm{er}}$ siècle avant notre ère (Carlson 2014, p. 55-56). L'Égée centrale constituerait ainsi une région privilégiée de réception des marchandises occidentales, italiques (de l'Adriatique majoritairement) mais également africaines, aux II ${ }^{\mathrm{e}}$ et $\mathrm{I}^{\mathrm{er}}$ siècles av. J.-C.

\section{- Les spécificités du Sud-Est égéen}

Pour M. Lawall la situation serait différente dans le Sud-Est égéen au sein duquel les productions amphoriques se sont particulièrement développées au début de la période hellénistique à Rhodes, Cos, Cnide (Lawall 2004, p. 275). À partir du milieu du $\mathrm{II}^{\mathrm{e}}$ siècle av. J.-C. les conditions favorables offertes aux marchands romains en Méditerranée orientale auraient profité aux marchandises occidentales, aux dépens des produits égéens, en particulier du vin. Cette concurrence nouvelle aurait provoqué un "effondrement » rapide de l'économie locale (Lawall 2004, p. 275). Celui-ci n'a peut-être pas été aussi prononcé et les réseaux commerciaux ont sans doute continué à fonctionner.

91 L'argumentation en faveur de cette " crise économique » repose sur l'idée qu'au-delà du milieu $d u \mathrm{II}^{\mathrm{e}}$ siècle les amphores, en particulier rhodiennes, ne bénéficiaient plus des débouchés fournis par les marchés extra-égéens (Lawall 2004, p. 275). Peut-être faut-il nuancer ce point. Les découvertes récentes faites en France à Toulouse sur le chantier de la Caserne Niel montrent que des amphores rhodiennes, associées aux phases 2 et $3 \mathrm{du}$ site, respectivement datées entre 160 et 140 av. J.-C. et 140 et 130 av. J.-C., ont été commercialisées en Gaule vers le milieu et dans la seconde moitié du II $^{\mathrm{e}}$ siècle avant notre ère (Loughton et Alberghi 2012, p. 806-807). Des découvertes plus anciennes témoignent également de la commercialisation vers la Gaule en faibles quantités d'amphores égéennes, de Rhodes mais également de Cos, sans doute via l'Italie, à la même période et encore dans le courant du $\mathrm{I}^{\mathrm{er}}$ siècle av. J.-C. (Lemaître 2002, p. 221 et 224). D'un point de vue quantitatif, ces exportations vers l'Ouest sont sans commune mesure avec les centaines de milliers d'amphores rhodiennes retrouvées en Méditerranée orientale, mais il nous semble qu'elles attestent, d'une certaine manière, de l'intégration des amphores produites dans les cités grecques du Sud-Est égéen dans les réseaux du grand commerce méditerranéen, entre la fin de la période hellénistique et les deux premiers siècles de l'Empire (Lemaître 2002).

Les découvertes d'amphores italiques en Lycie, intervenant entre le milieu du $\mathrm{II}^{\mathrm{e}}$ siècle av. J.-C. et se prolongeant au début de la période impériale semblent montrer, que la région a participé à la réception des denrées originaires de la façade adriatique et de la façade 
tyrrhénienne de l'Italie. Les faibles quantités connues à l'heure actuelle, soit une quarantaine d'exemplaires mise au jour dans l'ouest de la Lycie, sont sans doute en partie liées au caractère ponctuel des explorations archéologiques dans la région.

Pour autant, les arrivages de produits occidentaux en Lycie se faisaient surtout à la faveur du tracé des routes commerciales sillonnant le sud de la mer Égée via la Crète, Rhodes et la côte sud-anatolienne (et lycienne). Dans cette configuration de réseau de commercialisation, le recours au relais délien, n'était peut-être pas nécessaire et même inenvisageable après le deuxième sac de l'île en 69 av. J.-C.

Les lieux lyciens, dans lesquels les amphores italiques sont attestées, correspondent soit à des ports, comme Patara, soit à des centres urbains comme Xanthos en retrait par rapport au trait de côte, mais en relation avec le littoral via un fleuve, soit à des sanctuaires comme le Létôon de Xanthos, des sites dont la nature est propice aux séjours d'étrangers, marchands ou non. La fin de la tutelle rhodienne accordée par le sénat romain en 166 av. J.-C. et la reconnaissance manifestée par la Confédération lycienne ont sans doute créé des conditions propices à l'épanouissement des intérêts des hommes d'affaires italiens ou de leurs intermédiaires dans la région.

\section{Conclusion}

$95 \mathrm{Au}$ terme de cette présentation, plusieurs remarques complémentaires peuvent être formulées. Il est pour le moment difficile de mettre en relation les amphores italiques en Lycie avec la présence de marchands italiens dans un contexte politique et économique méditerranéen qui leur était favorable. Les données sont trop ténues. Ces arrivages de vins, d'huile et de sauces ou conserves de poisson, modestes et sans commune mesure avec les quantités observées à Délos ou Athènes, s'inscrivent dans le temps long, entre le milieu $d u{ }^{\mathrm{e}}{ }^{\mathrm{e}}$ siècle av. J.-C. et le début de l'époque impériale. Les conditions de consommation de ces denrées ne sont malheureusement pas connues. Seules les découvertes faites dans le sanctuaire du Létôon peuvent éventuellement être liées aux fêtes et cérémonies religieuses qui se déroulaient dans le sanctuaire et au cours desquelles le vin, l'huile étaient utilisés et consommés. L'inscription faisant état du catalogue des épreuves des Romaia, attachées au sanctuaire du Létôon, montre que le concours attirait aux côtés des Lyciens, des étrangers, grecs, athéniens, alexandrins et romains (Robert 1978, p. 279). Un Romain, installé à Telmessos, apparait d'ailleurs parmi les noms des vainqueurs aux épreuves hippiques (Robert 1978, p. 282).

Les indices de découvertes semblent dessiner, plutôt qu'un passage par Délos, une route privilégiant, l'acheminement des denrées italiques par le sud de la mer Égée. L'affranchissement des Lyciens de la tutelle rhodienne après 167 av. J.-C. ne signifie pas pour autant la fin des relations entre les deux. La Confédération lycienne était même en alliance militaire avec les Rhodiens au début $\mathrm{du} \mathrm{I}^{\mathrm{er}}$ siècle avant notre ère (Baker et Thériault 2005, p. 349).

97 Enfin, les observations préliminaires que nous avons pu faire sur les argiles employées par les potiers italiens semblent indiquer que les productions de la façade tyrrhénienne sont majoritaires en Lycie, d'autant plus si on avance dans le premier siècle de notre ère. L'étude de nouveaux ensembles d'amphores et la découverte de timbres sont indispensables pour étoffer ces premiers éléments. Malgré leur grande fragilité, ces données corroborent l'idée déjà ancienne de liens économiques privilégiés entre la 
Campanie et la Méditerranée orientale. Elles contribuent à mettre en lumière la commercialisation des produits italiques le long de la côte sud-anatolienne. Des hommes d'affaires, dont nous ignorons tout à ce stade de l'enquête ${ }^{17}$, agissant pour leur compte ou servant d'intermédiaire, ont investi le moindre port ou centre urbain de Méditerranée orientale, arrivant jusqu'en Lycie, pourtant réputée pour être un peu coupée du monde.

\section{BIBLIOGRAPHIE}

Alpözen T. O., Berkaya B. et Ödas A. (1995), Commercial amphoras of the Bodrum Museum of underwater Archaeology, Bodrum.

Andreau J. (1997), « Rôle de l'économie dans le passage de la République à l'Empire », dans Bruhns H., David J.-M., Nippel W. éd., Die späte römische Republik. La fin de la République romaine. Un débat franco-allemand d'histoire et d'historiographie, Rome, p. 169-184.

Arnaud P. (2005), Les routes de la navigation antique. Itinéraires en Méditerranée, Paris.

- (2011), « La Lycie et la Carie du Stadiasme », Anatolia Antiqua 19, p. 411-432.

Arthur P. et Oren D.E. (1998), « The North Sinai survey and the evidence of transport amphorae for the Roman and Byzantine patterns », JRA 11, p. 193-212.

Badie A., Lemaitre S. et Moretti J.-C. (2004), « Le théâtre du Létôon de Xanthos. État des recherches en 1999 », Anatolia Antiqua XII, p. 145-186.

Baker P. et Thériault G. (2005), « Les Lyciens, Xanthos et Rome dans la première moitié du $1^{\mathrm{er}} \mathrm{s}$. a. C. : nouvelles inscriptions », REG 118, p. 329-366.

Baudoux J. (1996),Les amphores du nord-est de la Gaule. Contribution à l'histoire de l'économie provinciale sous l'Empire romain, Paris.

Benquet L. et Piot C. (2000), « Les amphores de Lacoste (Mouliets-et-Villemartin, Gironde) », dans SFECAG, Actes du Congrès de Libourne, p. 155-165.

Bezeczky T. (2004), «Early Roman Food Import in Ephesus : Amphorae from the Tetragonos Agora ", dans Eiring J. et Lund J. éd., Transport Amphorae and Trade in the Eastern Mediterranean, Acts of the International Colloquium at the Danish Institute at Athens, September 26-29, 2002, Athènes, p. 85-97.

- (2006), « Consumer behaviour in Ephesus in the first century BC », dans Malfitana D., Poblome J. et Lund J. éd., Old Pottery in a new Century. Innovating perspectives on roman Pottery Studies. Atti des Convegno Internazionale di Studi Catania, 22-24 Aprile 2004, Catane, p. 287-308.

- (2010), «Italian Wine in the Eastern Mediterranean. Amphorae from Etruria, Latium, and Campania from the fourth century B.C. to the first century A.D. The Case of the Ephesian Amphorae ", dans XVII International Congress of Classical Archaeology, Roma 22-26 Sept. 2008, Bollettino di Archeologia On-Line I, volume speciale B, B8, 6, Rome.

- (2012), « Roman Amphorae in Ephesus », Forum Archaeologiae 62, III. URL : http:// homepage.univie.ac.at/elisabeth.trinkl/forum/forum0312/62amphora.htm 
Botte E. (2009), Salaisons et sauces de poissons en Italie du sud et en Sicile durant l'Antiquité, Collection du Centre Jean Bérard 31, Naples.

Böttger B. (1992), « Die kaiserzeitlichen und spätantiken Amphoren aus dem Kerameikos », MDAI (A) 107, p. 3-381, Taf. 96-102.

Bresson A. (1999), « Rhodes and Lycia in Hellenistic Times », dans Gabrielsen V., Bide P., Engbergpedersen T., Hannestad L. et Zahle J. éd., Hellenistic Rhodes. Politics, Culture and Society, Aarhus, p. 98-131.

- (2002), «Italiens à Rhodes et à Caunos ", dans Müller C. et Hasenohr Cl. éd., Les Italiens dans le monde grec (II ${ }^{e}$ s. av. J.-C./Ir s. ap. J.-C.) : circulation, activités, intégration. Actes de la table-ronde de l'École normale supérieure (Paris 14-16 mai 1998), BCH Suppl. 41, Athènes, p. 147-162.

Carlson D. (2014), « Dating a Shipwrecked Marble Cargo Destined for the Temple of Apollo at Claros », dans Moretti J.-C. éd. et Rabatel L. coll., Le sanctuaire de Claros et son oracle, Actes du colloque international de Lyon, 13-14 janvier 2012, Lyon, p. 51-61.

Cavalier L. (2003), « Nouvelles tombes de Xanthos », Anatolia Antiqua XI, p. 201-214. DOI : 10.3406/ anata.2003.1003

- (2012), «La basilique civile de Xanthos : étude architecturale et proposition de restitution », dans Cavalier L., Descat R. et des Courtils J. éd., Basiliques et agoras de Grèce et d'Asie Mineure, Bordeaux, p. 189-199.

Çevik N., Bulut S. et Akyürek E. (2012), « Excavations and Surveys at Myra-Andriake in 2011 », Anmed, p. 65-72.

Cipriano M.-T. et Carre M.-B. (1989), « Production et typologie des amphores sur la côte adriatique de l'Italie ", dans Amphores romaines et histoire économique. Dix ans de recherche. Actes du colloque de Sienne (22-24 mai 1986), Rome, p. 67-104.

des Courtils J. (2003), « Xanthos en Lycie : nouvelles données sur la romanisation d'une ancienne cité indigène », REG 116,1, p. 1-16.

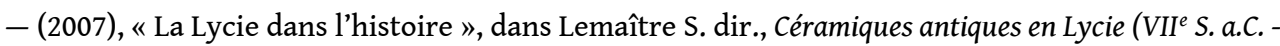
VII ${ }^{e}$ S. p.C.). Les produits et les marchés, Actes de la table-ronde de Poitiers, Bordeaux, p. 11-23.

Desbat A. (1998), « L'arrêt des importations de Dressel 1 en Gaule », SFECAG, Actes du congrès d'Istres, Marseille, p. 31-36.

Desbat A., Savay-Guerraz H. (1990), « Note sur la découverte d'amphores Dressel 2/4 italiques, tardives, à Saint-Romain-en-Gal (Rhône) », Gallia 47, p. 203-213.

Dressel H. (1899), Corpus Inscriptionum Latinarum, XV, 2.

Dündar E. (2013), « Roman Amphora Stamps from Patara », Olba XXI, p. 142-149.

Élaigne S. (2012), La vaisselle fine de l'habitat alexandrin. Contribution à la connaissance de la mobilité des techniques et des produits céramiques en Méditerranée du II siècle av. J.-C. à l'époque claudienne, Le Caire.

Empereur J.-Y. (1982), « Les anses d'amphores timbrées et les amphores, aspects quantitatifs », BCH 106, p. 219-233.

- (1983), « Appendice : les amphores », dans Bruneau P. et Ducat J. éd., Guide de Délos, Athènes, p. 97-98.

- (1986), « Les amphores de Délos », Dossiers Histoire et Archéologie 105, p. 67-69. 
- (1998), « Les amphores complètes du Musée d'Alexandrie : importations et productions locales ", Commerce et artisanat dans l'Alexandrie hellénistique et romaine, BCH Suppl. 33, p. 393-399. Empereur J.-Y., Braun J.-P., Le Bras H., Fraisse P. et Le Dinahet-Couilloud M.-T. (1983), « Délos et Rhénée ", BCH 107, 2, p. 881-899.

Empereur J.-Y., Hesnard A. (1987), « Les amphores hellénistiques », dans Lévêque P. et Morel J.-P., Céramiques hellénistiques et romaines, II, Besançon-Paris, p. 9-72.

Empereur J.-Y., Picon M. (1989), « Les régions de production d'amphores impériales en Méditerranée Orientale ", Amphores romaines et histoire économique, dix ans de recherches, Actes du colloque de Sienne, mai 1986, Rome, p. 223-248.

Étienne R. (2002), « Introduction », dans Müller C. et Hasenohr C. éd., Les Italiens dans le monde grec (II ${ }^{e}$ s. av. J.-C./I Ir S. ap. J.-C.) : circulation, activités, intégration. Actes de la table-ronde de l'École normale supérieure (Paris 14-16 mai 1998), BCH Suppl. 41, Athènes, p. 1-8.

Ferrazzoli A.F. et Ricci M. (2009), «Cilician ceramic production from the roman to byzantine Age. New data Elaiussa Sebaste », Olba, XVII, p. 33-44.

Finkielsztejn G. (2001), Chronologie détaillée et révisée des éponymes amphoriques rhodiens, de 270 à 108 av. J.-C. environ. Premier bilan, Oxford.

Formenti F., Hesnard A. et Tchernia A. (1978), « Note sur le contenu d'une amphore Lamboglia 2 de l'épave de la Madrague de Giens », Archaeonautica 2, p. 95-100.

Grace V. (1979), Amphoras and the ancient wine trade, Princeton.

Guichard V. (1997), « Les amphores », dans Lavendhomme M.-O. et Guichard V. éd. Rodumna (Roanne, Loire), le village gaulois, Paris, p. 133-143.

Guitton D., Lemaître S. avec la collaboration de Brunie D. et Maguer P. (2012), « Évolution des vaisseliers dans la vallée du Clain de la fin du $\mathrm{II}^{\mathrm{e}}$ s. av. J.-C. au début du $\mathrm{I}^{\mathrm{er}}$ s. ap. J.-C. : les exemples des sites des Gains à Saint-Georges-les-Baillargeaux et de la Z.A.C. Saint-Éloi à Poitiers (Vienne) », dans SFECAG, Actes du Congrès de Poitiers, p. 55-104.

Hatzfeld J. (1919), Les trafiquants italiens dans l'Orient hellénique, Paris.

Hayes J. W. (1985), « Sigillate orientali », Atlante delle forme ceramiche II, Ceramica fine romana nel bacino mediterraneo (tardo ellenismo e primo impero), Enciclopedia dell'arte antica classica e orientale, Rome, p. 1-95.

Hesnard A. (1977), « Note sur un atelier d'amphores Dr. 1 et Dr. 2-4 près de Terracine », MEFRA 89, p. 157-168.

Hesnard A., Carre M.-B., Rival M., Dangréaux B., Thinon M., Blaustein M., Dumontier, Chene A., Foliot P. et Bernard-Maugiron H (1988), « L'épave romaine Grand Ribaud D (Hyères, Var) », Archaeonautica 8, p. 5-180.

Hesnard A. et Lemoine Ch. (1981), « Les amphores du Cécube et du Falerne. Prospections, typologies, analyses », MEFRA 93, 1, p. 243-395.

Laubenheimer F. et Marlière E. (2010), Échanges et vie économique dans le Nord-Ouest des Gaules (Nord/Pas-de-Calais, Picardie, Haute-Normandie). Le témoignage des amphores du II ${ }^{e}$ s. av. J.-C. au IV $s$. ap. J.-C., Besançon.

Lawall M. (2006), « Consuming the West in the East: Amphoras of the western Mediterranean in the Aegean before $86 \mathrm{BC}$ », dans Malfitana D., Poblome J. et Lund J. dir., Old Pottery in a New 
Century: Innovating Perspectives on Roman Pottery Studies (Atti del convegno internazionale di studi, Catania, 22-24 Aprile 2004), Catane, p. 265-286.

Le Dinahet-Couilloud M.-T. (1974), Les monuments funéraires de Rhénée, Athènes.

Lemaître S. (2002), « Recherche sur la diffusion en Gaule, des amphores produites dans le sudouest de la Turquie, à l'époque impériale », dansBlondé F., Ballet P. et Salles J.-F., Céramiques hellénistiques et romaines. Productions et diffusion en Méditerranée orientale (Chypre, Égypte et côte syropalestinienne), Actes du colloque de Lyon (2-4 mars 2000), Lyon, p. 213-226.

- (2006), « La Lycie et les échanges commerciaux dans l'Antiquité : les données du mobilier amphorique. Étude préliminaire », dans Dörtlük K. et Varkivanç B. éd., Proceedings of The III ${ }^{\text {rd }}$ International Symposium on Lycia 07-10 november 2005, Antalya, p. 385-399.

- (2007a), « À propos des échanges en Syrie à l'époque romaine : les amphores de la fouille du centre-ville de Beyrouth Bey 002 ", dans Sartre M. éd., Productions et échanges dans la Syrie grecque et romaine, Actes de colloque, Université François Rabelais de Tours (juin 2003), Topoï Suppl. 8 , p. 271-295.

- (2007b), « La Lycie et les échanges commerciaux dans l'Antiquité. Les données du mobilier amphorique. Premiers éléments de réflexion », Recherches récentes sur la Lycie antique, Colloque International INHA (19 novembre 2005), Bulletin de la SFAC XXXVIII, 2005-2006, Revue Archéologique, 1, p. 175-180.

- (2012), avec la collaboration de Arqué M.-C., Auger A.-E. et Bidault J., « Pour qui sont ces amphores qui gisent sur vos sites. Importations méditerranéennes à Poitiers-Lemonum », dans SFECAG, Actes du Congrès de Poitiers, p. 181-195.

- (2014), «Les amphores (AM) », dans Deru X. dir., Durocortorum, la céramique de César à Clovis, Bulletin de la Société archéologique champenoise 107, p. 288-315.

- (à paraître), « Entre mers et déserts d'Égypte : sur la piste des amphores à vin italiques ", dans Sauzeau T. et Tallet G. éd., Mer et désert de l'Antiquité à nos jours, approches croisées, Colloque international de Limoges (7-9 novembre 2013), à paraître.

Lemaître S., Élaigne S. et Alary A. (2004), « Le mobilier céramique d'un remblai romain dans le sanctuaire du Létôon de Xanthos », Anatolia Antiqua XII, p. 329-333.

Lemaître S., Waksman S.Y., Arqué M.-C., Marksteiner B., Pellegrino E. et Rocheron C. (2013), «Identité régionale et spécificités locales en Lycie : l'apport des céramiques culinaires », dans Brun P., Cavalier L., Konuk K. et Prost F. éd., Euploia. La Lycie et la Carie antiques, Bordeaux, p. 189-212.

Loughton M. (2000), « La morphologie des amphores républicaines en Auvergne », dans SFECAG, Actes du Congrès de Libourne, Marseille, p. 243-256.

Loughton M. et Alberghi L. (2012), « Toulouse, "Caserne Niel" (Haute-Garonne) : a preliminary report on the amphorae ", dans SFECAG, Actes du Congrès de Poitiers, p. 801-820.

Lund J. (2000), « Transport Amphorae as Evidence of Exportation of Italian Wine and Oil to the Eastern Mediterranean in the Hellenistic Period », dans Lund J. et Pentz P. éd., Between Orient and Occident, Studies in Honour of P.J. Riis, Copenhague, p. 77-99.

Manacorda D. (1981), « Produzione agricola, produzione ceramica e proprietari nell'Ager Cosanus nel I secolo a.C. ", Società romana e produzione schiavistica II. Merci, mercato e scambi nel Mediterraneo, Rome-Bari, p. 3-54 et 263-273.

Marabini Moeus M. T. (1973), The Roman Thin Walled Pottery from Cosa (1948-1954), Rome. 
Maza G. (1998), « Recherche méthodologique sur les amphores gréco-italiques et Dressel 1 découvertes à Lyon, $\mathrm{II}^{\mathrm{e}}-\mathrm{I}^{\mathrm{er}}$ siècles avant J.-C. ", dans SFECAG, Actes du congrès d'Istres, Marseille, p. 11-29.

Menchelli S., Pasquinucci M., Capelli C., Cabella R. et Piaza M. (2008), « Anfore adriatiche nel Piceno meridionale », dans Rei Cretariae Romanae Acta 40, p. 379-392.

Metzger H. (1972), Les céramiques archaïques et classiques de l'acropole lycienne, Fouilles de Xanthos, IV, Paris.

Monsieur P., De Paepe P. (2002), « Amphores de Cos et amphores italiques à Pessinonte : croiser les données archéologiques et pétrographiques », Anatolia Antiqua, 10, p. 155-175.

Müller C. et Hasenohr Cl. éd. (2002), Les Italiens dans le monde grec (II ${ }^{e}$ s. av. J.-C./IIr s. ap. J.-C.) : circulation, activités, intégration. Actes de la table-ronde de l'École normale supérieure (Paris 14-16 mai 1998), BCH Suppl. 41, Athènes.

Olmer F. (2003), Le commerce du vin chez les Éduens d'après les timbres d'amphores, Bibracte 7, Gluxen-Glenne.

- (2012), « Les amphores sont-elles utiles à la chronologie de la fin de l'âge du Fer ? », dans Barral P. et Fichtl S. dir., Regards sur la chronologie de la fin de l'âge du Fer (III ${ }^{e}-I^{\text {er }}$ siècle avant notre ère) en Gaule non méditerranéenne, Actes de la Table-ronde tenue à Bibracte (15-17 octobre 2007), Bibracte 22, p. 273-300 et annexes numériques 1 à 22 .

Poux M. (2004), L'Âge du vin. Rites de boisson, festins et libations en Gaule indépendante, Protohistoire Européenne 8, Montagnac.

Poux M. et Selles H. (1998), «Vin italique en pays carnute. À propos d'un lot d'amphores Dressel 1 découvert à Chartres, rue Sainte-Thérèse ", dans SFECAG, Actes du Congrès d'Istres, Marseille, p. 207-224.

Rauh N.K. (1999), « Rhodes, Rome and the Eastern Mediterranean Wine Trade, 166-88 BC », dans Gabrielsen V., Bilde P., Engberg-Pedersen T., Hannestad L. et Zahle J. éd., Hellenistic Rhodes : Politics, Culture, and Society, Studies in Hellenistic Civilization IX, Aarhus, p. 162-186.

- (2003), Merchants, Sailors and Pirates in the Roman World, Stroud-Gloucestershire.

Robert L. (2/1978), « Catalogue agonostique des Romaia de Xanthos », Revue Archéologique, fasc. 2, p. 277-290.

Rocheron C. (2011), La céramique commune à pâte calcaire de Xanthos et du Létôon de l'époque classique à la période protobyzantine, thèse de doctorat de l'Université Bordeaux 3, Bordeaux.

Şenol A. K. (2007), « A statistical Essay on the Distribution of Imported Amphorae Finds of the CEAlex Salvage Excavations ", dans Marchand S. et Marangou A. éd., Amphores d'Égypte de la Basse Époque à l'époque arabe, Cahiers de la Céramique Égyptienne 8, vol. I, p. 57-75.

şenol A. K. (2009), Taşucu Arslan Eyce Amphora Müzesi, Silifke.

Stöckli W. (1979), Die Grob- und Importkeramik von Manching, Ausgrabungen in Manching, Band 8, Wiesbaden.

Tchernia A. (1986a), Le vin de l'Italie romaine, BEFAR 261, Rome.

- (1986b), « Amphores et textes : deux exemples », dans Empereur J.-Y. et Garlan Y. éd., Recherches sur les amphores grecques, BCH Suppl. 13, p. 31-36.

Vandermersch C. (1994), Vins et amphores de Grande Grèce et de Sicile IV -III $^{e}$ s. avant J.-C., Naples.

Will E.L. (1982), « Greco-italic Amphoras », Hesperia 51, p. 338-356. 
- (1989), «Relazioni mutue tra le anfore romane. I retrovamenti in oriente, alla luce dei dati ottenuti nell'occidente ", dans Amphores romaines et histoire économique : dix ans de recherche. Actes du colloque (Sienne. 22-24 mai 1986), Rome, p. 297-309.

- (1997), « Shipping amphoras as indicators of economic romanization in Athens », dans Hoff M. C. et Rotroff S. I. éd., The Romanization of Athens : Proceedings of an International Conference held at Lincoln, Nebraska (April 1996), Oxford, p. 117-133.

Williams D.F. (2004), « The Eruption of Vesuvius and its Implications for the Early Roman Amphora Trade with India ", dans Eiring J. et Lund J. éd., Transport Amphorae and Trade in the Eastern Mediterranean, Acts of the International Colloquium at the Danish Institute at Athens, September 26-29, 2002, Athènes, p. 441-450.

\section{NOTES}

1. Les amphores ovoïdes produites dans le sud de la péninsule italienne semblent s'inscrire dans un groupe plus large de conteneurs à la silhouette comparable fabriqués également en différents points de la péninsule ibérique et en Afrique et qui fait l'objet de recherches nouvelles. Cf. sur ce sujet le workshop international organisé à Séville en décembre 2015: A family Business... The ovoid Amphorae in the Central and Western Mediterranean. Between the last two centuries of the Republic and the early days of the Roman Empire, sous la direction de E. García Vargas, R. R. de Almeida, H. González Cesteros et A. M. Sáez Romero.

2. Observation personnelle de l'auteure à l'occasion d'une mission d'étude en Cilicie en août 2012, dans le cadre du programme franco-allemand Xanthiaca financé par l'ANR.

3. Ces travaux se sont déroulés dans le cadre des recherches menées par l'équipe de la mission française de Xanthos-Létôon à laquelle nous avons été intégrée de 1995 à 2010, moment où la mission française a été suspendue par les autorités turques.

4. Nous remercions chaleureusement L. Cavalier d'avoir accepté que nous publiions ici la coupe stratigraphique du sondage 14050 et $\mathrm{E}$. Cayre pour le document graphique.

5. Les différentes mesures réalisées sur les fragments ont été faites en suivant les préconisations présentées dans Maza 1998, p. 12, fig. 2.

6. La question du contenu des amphores fait l'objet de nouveaux développements liés au progrès des méthodes d'analyses chimiques et paléogénomiques d'échantillons provenant des parois internes des conteneurs. Cf. Roman Amphora Contents reflecting on maritime Trade of food stuffs in Antiquity. International Interactive Conference (5-7 october 2015), Cadiz, D. Bernal Casasola, M. Bonifay et A. Pecci (org.).

7. Ces éléments proviennent de la base de données réalisée par C. Rocheron dans le cadre de ses recherches sur les mobiliers céramiques de Xanthos. Nous la remercions pour la communication de ces informations.

8. Nous avons pu faire ces observations sur place au moment de l'étude des amphores à Xanthos.

9. Étude inédite M.-C. Arqué.

10. Les mobiliers mis au jour dans ce remblai seront publiés dans le cadre d'un ouvrage collectif actuellement en préparation: S. Lemaître (dir.), Cuisiner, boire et manger dans le sanctuaire du Létôon à l'époque impériale. 
11. Nous remercions notre collègue $E$. Dündar d'avoir attiré notre attention sur cette publication récente.

12. Une première présentation des résultats obtenus dans le cadre de ce programme de prospection a été donnée par B. Yener-Marksteiner et l'auteur de ces lignes à Poitiers en octobre 2014, lors du colloque international Les mobiliers archéologiques dans leur contexte de découverte. Fonctions et statuts et dont la publication des actes est en cours de préparation.

13. La proposition de rapprochement des exemplaires découverts à Xanthos avec des productions de Campanie repose sur l'observation macroscopique de critères caractéristiques des pâtes des amphores produites dans les ateliers de la région, établis à partir d'échantillons d'amphores provenant des ateliers campaniens analysés et conservés au laboratoire de céramologie de Lyon, ARAR, UMR 5138. Toutefois, des analyses pétrographiques et chimiques seraient nécessaires pour confirmer l'origine campanienne des conteneurs italiques mis au jour à Xanthos.

14. Observation personnelle lors d'une mission en Cilicie en 2012 dans le cadre du programme Xanthiaca financé par l'ANR.

15. Exposées en 2012 dans le musée de M. Arslan Eyce.

16. Cf. développement ci-dessus $\S 2$.

17. Le recoupement des données épigraphiques concernant les Italiens présents en Lycie et plus largement dans le Sud-Ouest anatolien avec celles livrées par les timbres sur amphores italiques constitue l'étape suivante de notre travail. Il permettra peut-être de commencer à formuler une réponse à la question posée par $\mathrm{N}$. Tran lors de la rencontre de Poitiers à propos des «élites négociantes »!

\section{RÉSUMÉS}

L'activité des marchands au long cours sillonnant l'espace méditerranéen d'Ouest en Est a depuis les premières décennies $\mathrm{du} \mathrm{xx}^{\mathrm{e}}$ siècle suscité l'intérêt des historiens. L'analyse des sources littéraires et épigraphiques montre que la présence des Italiens dans le bassin oriental de la Méditerranée s'est notablement accrue à partir du $\mathrm{II}^{\mathrm{e}}$ siècle av. notre ère. Les deux derniers siècles de la République sont également marqués par la mise en oeuvre par Rome d'une politique hégémonique en Orient. La découverte, dans de nombreux sites de cette région, d'amphores produites sur la façade adriatique et sur la côte tyrrhénienne de l'Italie tend à étayer l'hypothèse de la constitution de réseaux commerciaux destinés à l'exportation vers l'est des denrées agricoles italiennes, vin et huile prioritairement. La cité de Xanthos a livré un ensemble de mobiliers céramiques associé aux remblais de construction d'un portique bordant l'agora romaine du site, composé notamment d'un lot d'amphores égéennes et italiques. Cet assemblage permet d'insérer Xanthos et plus largement la Lycie dans les réseaux de commercialisation des produits italiques qui ont touché le bassin oriental de la Méditerranée aux $\mathrm{II}^{\mathrm{e}}$ et $\mathrm{I}^{\mathrm{er}}$ siècles avant notre ère.

The activity of merchants crisscrossing the mediterranean Sea from West to East has since the early decades of the twentieth century attracted the attention of historians. The analysis of 
literary and epigraphic sources shows that the presence of Italians in the Eastern increased significantly from the second century BC. The last two centuries of the Republic are also marked by the implementation by Rome of a hegemonic policy in the East. The discovery in many sites in this region, amphoras produced on the Adriatic and the Tyrrhenian coast of Italy tends to support the hypothesis of the constitution of commercial networks for export to the East, of italian agricultural products, wine and oil primarily. In Lycia, the city of Xanthos has delivered ceramics assemblages associated with the fill related to a portico bordering the Roman Agora on the site. These level was composed of aegean and italic amphoras. This archaeological context gives the opportunity to insert Xanthos and widely Lycia in marketing italics products networks that hit the eastern part of the Mediterranean to the second and first centuries BC.

INDEX

Mots-clés : Lycie, amphore italique, navigation, réseaux commerciaux, marchand

Keywords : Lycia, italic amphora, networks exchange, merchant

\section{AUTEUR}

\section{SÉVERINE LEMAÎTRE}

EA 3811 HeRMA, UFR Sciences Humaines et Arts, Université de Poitiers 